\title{
Fluid displacement between two parallel plates: a non-empirical model displaying change of type from hyperbolic to elliptic equations
}

\author{
By M. SHARIATI $I^{1} \dagger$, L. TALON $N^{2}$, J. MARTIN ${ }^{2}$, \\ N. RAKOTOMALALA ${ }^{2}$, D. SALIN ${ }^{2}$ AND Y. C. YORTSOS ${ }^{1} \ddagger$ \\ ${ }^{1}$ Department of Chemical Engineering, University of Southern California, Los Angeles, \\ CA 90089-1211, USA \\ ${ }^{2}$ Laboratoire Fluides Automatique et Systèmes Thermiques, Universités P. et M. Curie and Paris Sud, \\ CNRS (UMR 7608) Bâtiment 502, Campus Universitaire, 91405 Orsay Cedex, France
}

(Received 28 October 2003 and in revised form 28 June 2004)

We consider miscible displacement between parallel plates in the absence of diffusion, with a concentration-dependent viscosity. By selecting a piecewise viscosity function, this can also be considered as 'three-fluid' flow in the same geometry. Assuming symmetry across the gap and based on the lubrication ('equilibrium') approximation, a description in terms of two quasi-linear hyperbolic equations is obtained. We find that the system is hyperbolic and can be solved analytically, when the mobility profile is monotonic, or when the mobility of the middle phase is smaller than its neighbours. When the mobility of the middle phase is larger, a change of type is displayed, an elliptic region developing in the composition space. Numerical solutions of Riemann problems of the hyperbolic system spanning the elliptic region, with small diffusion added, show good agreement with the analytical outside, but an unstable behaviour inside the elliptic region. In these problems, the elliptic region arises precisely at the displacement front. Crossing the elliptic region requires the solution of essentially an eigenvalue problem of the full higher-dimensional model, obtained here using lattice BGK simulations. The hyperbolic-to-elliptic change-of-type reflects the failing of the lubrication approximation, underlying the quasi-linear hyperbolic formalism, to describe the problem uniformly. The obtained solution is analogous to non-classical shocks recently suggested in problems with change of type.

\section{Introduction}

Moving fronts are encountered in many processes in science and engineering. Often, the problems involve geometries where the length scale in the direction of the displacement $x$ is much larger than in the other two directions, $y, z$ (large aspect ratio). Then, the front moves along the $x$-direction, but in general has a three-dimensional structure. Typically, fronts are driven from a contrast in properties between the two far-field states (the 'upstream' and the 'downstream' state), that renders unstable states uniform in the $(y, z)$-coordinates. Fronts reflect a balance between externally forced instability and fine-scale dissipation, and in many aspects they denote self-similar asymptotic states (Barenblatt 1996). For example, this is the

$\dagger$ Present address: Applied Biosystems, Foster City, CA, USA.

$\ddagger$ Author to whom correspondence should be addressed: yortsos@usc.edu. 
case with fluid displacements in constricted geometries, or in porous media, in the flow of suspensions, in combustion, and in a number of other applications (Batchelor \& Janse van Rensburg 1985; Pelcé 1988; Lake 1989; Aldushin \& Matkowsky 1998).

In many situations, it is common to seek an upscaled (large-scale) formulation, where the displacement is expressed in terms of averaged quantities. Because of its travelling-wave nature, the resulting formulation is typically in terms of a quasi-linear hyperbolic system,

$$
\frac{\partial \boldsymbol{C}}{\partial t}+\frac{\partial \boldsymbol{F}(\boldsymbol{C})}{\partial x}=\mathbf{0}
$$

where $\boldsymbol{C}$ is an averaged concentration (in general, a vector of size $N$ ), $\boldsymbol{F}$ is an averaged flux (or 'fractional flow') function (a vector of size $N$ ) and $t$ and $x$ are appropriately rescaled time and distance, respectively. For example, in the flow of three phases in a porous medium, $\boldsymbol{C}=\left\{C_{1}, C_{2}\right\}$, where $C_{i}$ is the volumetric fraction of phase $i$. A large number of well-known processes are described by quasi-linear hyperbolic systems. From a long list, we cite classical applications in gas dynamics (Courant \& Friedrichs 1948), non-classical extensions to the flow of van der Waals gases (Truskinovsky 1987; LeFloch 2002), real materials (Menikoff \& Plohr 1989) and elastodynamics (Shearer \& Yang 1995).

Deriving the functional dependence of $\boldsymbol{F}(\boldsymbol{C})$ in (1), or in other quasi-linear formalisms, is based on the following argument. The quasi-linear system must also apply to the case of a uniform ('equilibrium') state, for example the steady-state, where the variables are independent of $t$ and $x$. Under these conditions, a corresponding 'equilibrium' fractional flow function, denoted as $\boldsymbol{F}_{p f}(\boldsymbol{C})$ (corresponding to parallel flow in our case of interest), can be derived. Then, for self-consistency, we must make in (1) the identification

$$
\boldsymbol{F}(\boldsymbol{C})=\boldsymbol{F}_{p f}(\boldsymbol{C})
$$

This approach underlies the formulation of various quasi-linear hyperbolic systems. For example, in the case of van der Waals gases (Truskinovsky 1987; LeFloch 2002; and references therein), the equilibrium assumption is the van der Waals equation of state. In the problem of elastodynamics (Shearer \& Yang 1995), it is the equilibrium (quasi-static) stress-strain relation. In flow in porous media, it is the quasi-static representation of flow using steady-state relative permeabilities (Lake 1989). In the context of the present paper, therefore, the 'equilibrium' state corresponds to pressure profiles independent of the transverse direction, and was denoted by Yang \& Yortsos (1997, referred to hereinafter as YY), as the 'parallel flow' approximation.

Quasi-linear hyperbolic systems often display a change of type, namely the development of an elliptic region. Because of their mathematical interest, these mixed hyperbolic-elliptic problems have been studied at length in the literature (Bell, Trangenstein \& Shubin 1986; Keyfitz 1989; Shearer \& Trangenstein 1989; Isaacson, Marchesin \& Plohr 1990; Jackson \& Blunt 2002; Juanes \& Patzek 2003). Batchelor \& van Rensburg (1985) derived the analogue of (1) in the context of sedimentation of bi-disperse suspensions, where the state variables are the volumetric fractions of the two different-size particles, and the flux functions depend only on the two volumetric fractions. The authors showed that uniform states within the elliptic region are unstable, and used the change of type in order to classify stable and unstable suspension flows. An analogous approach was used by Bell et al. (1986) in three-phase flow in porous media.

Stable solutions in a mixed-type problem can develop through the addition of sufficiently strong regularizing diffusion (or viscosity, in the context of gas dynamics), 
so that the solution path avoids the elliptic region (see below and also Jackson \& Blunt 2002). However, instability will persist when diffusion is small, as in the present paper. In general, the change of type cannot be handled analytically based on (1) alone, and various alternative methods have been proposed (e.g. Bell et al. 1986; Keyfitz 1989). Recent works have shown that in crossing the elliptic region, classical shocks, namely those based on the addition of small diffusion, cannot resolve the problem. Rather, additional physics must be included in the shock region, e.g. through terms of higher-order than diffusion, for example an effective capillarity (LeFloch 2002). These result in additional constraints, leading to non-classical shocks (LeFloch 2002). Progress has been hampered because often, models displaying change of type have been based on empirical flux functions. In fact, some authors have argued that the existence of a mixed-type region should be used as a criterion to reject the empirical model on which it is based, and to replace it by another (also empirical), for which an elliptic region does not develop (Fayers \& Matthews 1984; Juanes \& Patzek 2003). A more rigorous physical model will help to elucidate these issues. We will show below that such a model can indeed be obtained by considering fluid displacement in the gap of a Hele-Shaw cell (or equivalently, in a long capillary, although this will not be discussed here). This forms one of the objectives of this paper.

Past investigations on flows in Hele-Shaw geometries include the seminal works of Park \& Homsy (1984) and Reinelt \& Saffman (1985) for immiscible displacement, and Petitjeans \& Maxworthy (1996), Chen \& Meiburg (1996), Rakotomalala, Salin \& Watzky (1997), Lajeunesse et al. (1999) and Yang \& Yortsos (1997) for miscible displacement. The latter authors used an asymptotic formalism, based on the lubrication approximation and in the absence of diffusion, to describe an equivalent 'two-fluid' problem between two parallel plates. We used quotation marks above to indicate that in this approach no capillary forces are involved, and the problem solved is kinematic. The injected fluid (local dimensionless concentration $c=1$, viscosity $\mu_{1}$ ) was taken as one of the two fluids, the initial fluid (local dimensionless concentration $c=0$, viscosity $\mu_{0}$ ) being the other. In the absence of diffusion, the fluids do not mix, thus retaining their identity. Under the further assumptions that the resulting profile is symmetric and that the flow is uni-directional, YY derived a hyperbolic equation for the transverse-averaged concentration $C \equiv \int_{0}^{1} c \mathrm{~d} y$, reading as

$$
\frac{\partial C}{\partial t}+\frac{\partial F(C)}{\partial x}=0,
$$

where, the fractional flow function is exact,

$$
F(C)=\frac{C\left[3+(2 M-3) C^{2}\right]}{2\left[1+(M-1) C^{3}\right]},
$$

and depends only on the dimensionless mobility ratio $M \equiv \mu_{0} / \mu_{1}$ between initial and injected viscosities (inverse mobilities).

YY solved a Riemann problem with initial state $C=0$ and injection state $C=1$. When $M<3 / 2$, the function $F(C)$ does not contain an inflection point, it is convex downwards, and the solution is a rarefaction wave, with the tip velocity (the velocity of the concentration $C=0$ ) being the maximum velocity of the Poiseuille profile (equal to $3 / 2$ in the present notation). The accuracy of the solution was confirmed by lattice BGK (Bhatnagar-Gross-Krook) numerical simulations (Rakotomalala et al. 1997), which solve the full problem without making the potentially restrictive parallel-flow approximation. On the other hand, when $M>3 / 2$, the fractional flow function contains an inflection point. The solution of the same Riemann problem 


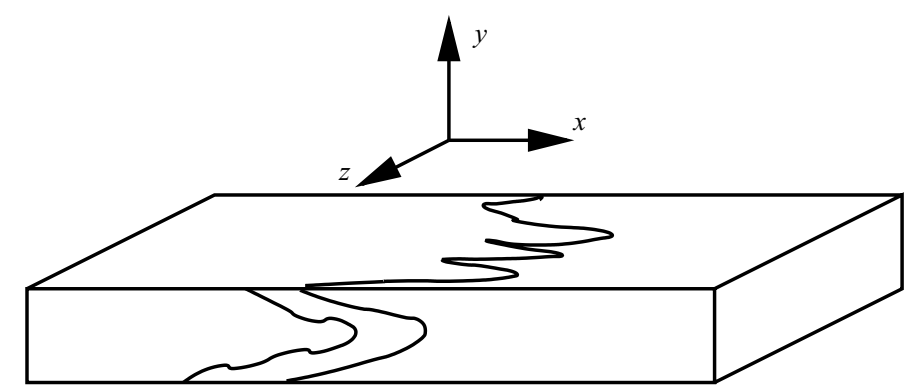

FIGURE 1. Schematic of the flow geometry for displacement in the gap of a Hele-Shaw cell.

involves now a contact discontinuity shock that precedes the spreading wave, with a shock velocity that exceeds the Poiseuille maximum and increases monotonically with the mobility ratio $M$. Under these conditions, the same numerical simulations (see also Chen \& Meiburg 1996) showed a discrepancy with the classical analysis, however, in that the front actually moves slower than predicted by the hyperbolic formalism, while its velocity becomes bounded at large $M$.

In retrospect, it is not surprising that the two solutions are different. In the vicinity of a shock, where transverse flow is significant, the parallel-flow formalism breaks down. Determining the correct front velocity there requires finding the eigenvalue of the full higher-dimensional problem. Viewed in a different context, it appears that the shock in YY belongs to the class of non-classical shocks. In a way, this is akin to the solvability question of the Saffman-Taylor problem (Saffman \& Taylor 1958; Pelcé 1988). An analytic determination of this problem, here in the context of the biharmonic, rather than the Laplace equation, remains open at present.

Motivated by the above, we will consider in this paper extending the YY approach to 'three-fluid' flow in the same geometry (figure 1). For this, we will take a piecewise mobility-concentration function, so that constant mobilities (or viscosities) are assigned to three different concentration (or fluid) regions, e.g. $M$ in $c_{a}<c<1$, $\Lambda_{m}$ in $c_{b}<c<c_{a}$, and 1 in $0<c<c_{b}$, and consider the evolution of the boundaries that separate these regions of constant mobility. As in YY, we will use the Stokes equations and the parallel-flow approximation in a symmetric profile across the gap. As we noted above, this assumption is necessary for self-consistency of the quasi-linear formalism and represents the equivalent of the equilibrium assumption, e.g. in gas dynamics. With these assumptions, we will show that the evolution of the corresponding volume fractions is described by a system of two quasi-linear hyperbolic equations, the classical solution of which is obtained by standard tools (Whitham 1976). More importantly, we will show that for a certain combination of parameters, the problem changes type and develops an elliptic region. The paper elaborates on the development and the analysis of corresponding Riemann problems, and provides a comparison with standard approaches. Crossing the elliptic region is addressed by solving the full higher-dimensional problem, using the lattice BGK method. Thus, based on an exact model in the absence of empiricism, but using specific assumptions, this paper presents evidence that change-of-type behaviour can indeed arise from real physical situations, but resolving it necessitates incorporating additional physics in the shock that spans the elliptic region, effectively solving an eigenvalue problem.

The paper is organized as follows. First, we provide the mathematical formulation, where the conditions for the development of mixed-type behaviour are outlined. Three 


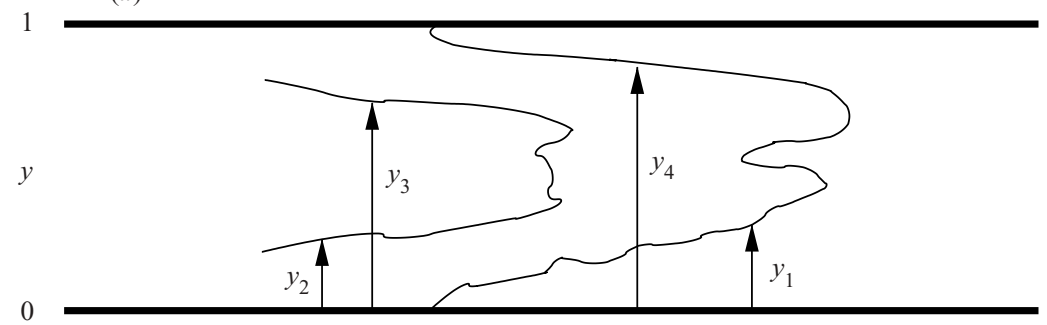

(b)

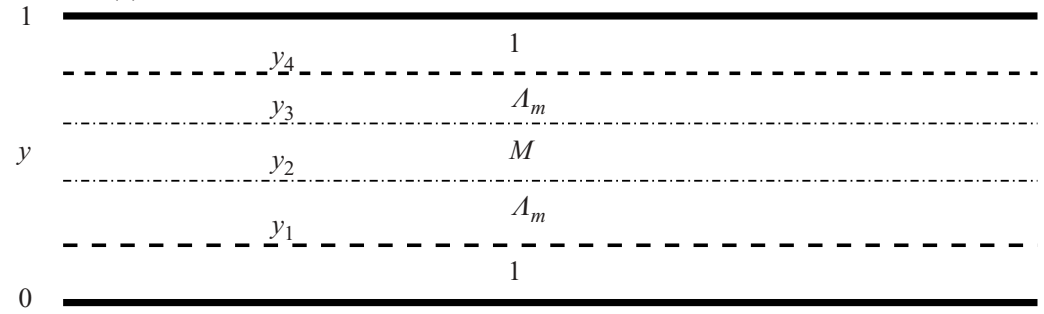

FiguRE 2. Schematic of the profiles assumed: $(a)$ general phase (finger) boundaries, $(b)$ corresponding mobility profile. The region near the wall has mobility 1 , boundaries $0<y<y_{1}(x, t)$ and $y_{4}(x, t)<y<1$, and it is denoted by subscript $w$; the region in-between has mobility $\Lambda_{m}$, boundaries $y_{1}(x, t)<y<y_{2}(x, t)$ and $y_{3}(x, t)<y<y_{4}(x, t)$, and it is denoted by subscript $o$; and the region in the middle of the domain has mobility $M$, boundaries $y_{2}(x, t)<y<y_{3}(x, t)$, and it is denoted by subscript $g$.

different, generic cases are analysed. Then, two specific Riemann problems are solved for a representative mobility profile. Numerical results based on the assumption of parallel flow, but with the presence of a small amount of diffusion, are subsequently described using lattice BGK simulations and compared to the analytical results. It is shown that unless diffusion is large, the diffusion-augmented models do not provide a physical solution in the mixed-type case. There, the full higher-dimensional model must be simulated. Discussion and conclusions follow.

\section{Mathematical formulation}

Consider miscible displacement in the gap of a Hele-Shaw cell in the $(X, Y)$-plane and in the absence of gravity, as shown in figure 1 . The cell, of thickness $H$, is initially saturated with a resident fluid (of dimensionless concentration $c=0$ ). Assume that a fluid with different concentration is injected at the constant rate $q$ to displace the fluid in place. The viscosity is a function of concentration (monotonic or nonmonotonic). A non-monotonic dependence is necessary for change-of-type behaviour, as will be shown below. It is worth noting that non-monotonic viscosity profiles in porous media flows have led to interesting results (e.g. see Manickam \& Homsy 1993; Loggia, Rakotomalala \& Salin 1999; Shariati 2000). However, the latter used a volume-averaged version of the problem, based on Darcy rather than Stokes equations.

In general, as the concentration field evolves, we can delineate three fluid regions (figure 2): a region near the wall, with mobility 1 and boundaries $0<y<y_{1}(x, t)$ and $y_{4}(x, t)<y<1$, denoted by subscript $w$; a region in-between with mobility $\Lambda_{m}$ and boundaries $y_{1}(x, t)<y<y_{2}(x, t)$ and $y_{3}(x, t)<y<y_{4}(x, t)$, denoted by subscript $o$; and a region in the middle of the domain, with mobility $M$ and boundaries 
$y_{2}(x, t)<y<y_{3}(x, t)$, denoted by subscript $g$. Because of the assumed absence of diffusion and the ensuing kinematic description, the fluids retain their mobility in their respective regions. Figure 2 can be viewed as the developed profile in a variable mobility displacement, where a fluid of mobility $M$ displaces one of initial mobility 1 , between which there is an intermediate fluid of mobility $\Lambda_{m}$. It also pertains to the case where two different fluids are injected at the inlet, each occupying a specific part of the inlet boundary. This coarse analogy will be used below to connect with the much studied problem of three-phase flow in a porous medium (Isaacson et al. 1990; Jackson \& Blunt 2002; Juanes \& Patzek 2003). The corresponding dimensionless mobility profile is stepwise,

$$
\begin{aligned}
\lambda= & 1+\left(\Lambda_{m}-1\right)\left[H\left(y-y_{1}(x, t)-H\left(y-y_{2}(x, t)\right)\right]\right. \\
& +\left(M-\Lambda_{m}\right)\left[H\left(y-y_{3}(x, t)-H\left(y-y_{4}(x, t)\right)\right],\right.
\end{aligned}
$$

where $H(z)$ is the Heaviside step function.

In dimensionless notation, the governing equations are

$$
\begin{gathered}
\frac{\partial c}{\partial t}+\frac{\partial(u c)}{\partial x}+\frac{\partial(w c)}{\partial y}=N_{T D}\left[\epsilon^{2} \frac{\partial^{2} c}{\partial x^{2}}+\frac{\partial^{2} c}{\partial y^{2}}\right], \\
\frac{\partial u}{\partial x}+\frac{\partial w}{\partial y}=0,
\end{gathered}
$$

where parameter $\epsilon=H / L$ is the aspect ratio, $x$ and $y$ have been scaled with the length and thickness, $L$ and $H$, of the cell, respectively, and for completeness we have retained diffusion, $N_{T D}=D L / q H^{2}$, where $D$ is molecular diffusivity. Using the analysis in YY (see also Shariati \& Yortsos 2001), and assuming a large aspect ratio, the parallel flow (equilibrium) expression for the velocity $u$ is

$$
u=\frac{G(y ; \lambda)}{\int_{0}^{1} G \mathrm{~d} y},
$$

where the function $G$ is

$$
G(y ; \lambda) \equiv \int_{0}^{y} \lambda \mathrm{d} y \int_{y}^{1} \lambda y \mathrm{~d} y-\int_{0}^{y} \lambda y \mathrm{~d} y \int_{y}^{1} \lambda \mathrm{d} y .
$$

As expected, in the passive tracer limit $\lambda=1$, we recover the Poiseuille velocity profile $u=6 y(1-y)$.

In the absence of diffusion, we can recast the mass balances by multiplying (6) by $\lambda^{\prime}$, where the prime denotes derivative with respect to $c$, to obtain

$$
\frac{\partial \lambda}{\partial t}+\frac{\partial(\lambda u)}{\partial x}+\frac{\partial(\lambda w)}{\partial y}=0 .
$$

Using the step mobility profile of (5), we can integrate (10) across the boundaries $y_{i}$, to obtain differential equations for their evolution. It is not difficult to show that the following equations result

$$
\frac{\partial y_{i}}{\partial t}+\frac{\partial}{\partial x}\left[\int_{0}^{y_{i}} u \mathrm{~d} y\right]=0 \quad(i=1,4) .
$$

These are coupled to each other through the integral term $G$ (equation (9)), from the parallel-flow approximation. To proceed, we will assume from now on that the profiles are symmetric across the gap. 
The symmetric case is described by $y_{4}(x, t)=1-y_{1}(x, t)$ and $y_{3}(x, t)=1-y_{2}(x, t)$, thus, by a system of two unknowns. For convenience, we will introduce the 'saturation' notation, $S_{w}=2 y_{1}, S_{g}=1-2 y_{2}$ and $S_{o}=1-S_{w}-S_{g}$, and the 'fractional flow' notation, $f_{w}=2 \int_{0}^{y_{1}} u \mathrm{~d} y, f_{g}=1-2 \int_{0}^{y_{2}} u \mathrm{~d} y$ and $f_{o}=1-f_{w}-f_{g}$, in analogy with the threephase flow problem. In terms of these variables, (11) takes the final conservation form

$$
\frac{\partial S_{w}}{\partial t}+\frac{\partial}{\partial x}\left[f_{w}\left(S_{w}, S_{g}\right)\right]=0
$$

and

$$
\frac{\partial S_{g}}{\partial t}+\frac{\partial}{\partial x}\left[f_{g}\left(S_{w}, S_{g}\right)\right]=0
$$

where we have the following explicit expressions for the fractional flow functions

$$
f_{w}=\frac{S_{w}^{2}\left(3-S_{w}\right)}{2\left[1+\left(\Lambda_{m}-1\right)\left(1-S_{w}\right)^{3}-\left(\Lambda_{m}-M\right) S_{g}^{3}\right]}
$$

and

$$
f_{g}=\frac{S_{g}\left[3+3\left(\Lambda_{m}-1\right)\left(1-S_{w}\right)^{2}+S_{g}^{2}\left(2 M-3 \Lambda_{m}\right)\right]}{2\left[1+\left(\Lambda_{m}-1\right)\left(1-S_{w}\right)^{3}-\left(\Lambda_{m}-M\right) S_{g}^{3}\right]} .
$$

These expressions are exact, in the context of the parallel-flow approximation. The properties $f_{w}\left(S_{w}=0, S_{g}\right)=0, f_{g}\left(S_{w}, S_{g}=0\right)=0, f_{w}\left(S_{w}=1, S_{g}\right)=1$ and $f_{g}\left(S_{w}, S_{g}=1\right)=1$, and the low saturation scalings, $f_{w} \sim S_{w}^{2}, f_{g} \sim S_{g}$ and $f_{o} \sim S_{o}$ demonstrate physical consistency with the three-phase flow analogy stated above. As expected, in the case $\Lambda_{m}=M$, the problem reduces to the 'two-fluid' flow problem of YY, since (14) is only a function of $S_{w}$ (compare with (4) and take $C=1-S_{w}$ ).

The derivation of (12)-(15) can also be obtained alternatively, by assuming piecewise constant concentration profiles in the three regions, postulating mass conservations for the two fluids and making use of the parallel-flow assumption (e.g. see Lajeunesse et al. 1999). Potential problems associated with the step or Dirac delta functions used in our approach will be resolved in shock or elliptic regions, in which additional physics that regularize the problem, and which are absent from the above, must be added, as noted above and also discussed below. The above kinematic description was shown to describe well away from shocks, actual experiments at high rates (see Lajeunesse et al. 1999).

The set of the two equations (12) and (13) form the quasi-linear system

$$
\frac{\partial \boldsymbol{u}}{\partial t}+\boldsymbol{A}(\boldsymbol{u}) \frac{\partial \boldsymbol{u}}{\partial x}=\mathbf{0},
$$

where $\boldsymbol{u}$ is the vector of the dependent variables,

$$
\boldsymbol{u}=\left[\begin{array}{l}
S_{w} \\
S_{g}
\end{array}\right]
$$

and $\boldsymbol{A}$ the $2 \times 2$ coefficient matrix

$$
\boldsymbol{A}=\left|\begin{array}{ll}
q_{1} & q_{2} \\
q_{3} & q_{4}
\end{array}\right|
$$

where

$$
\begin{array}{ll}
q_{1}=\frac{\partial f_{w}}{\partial S_{w}}, & q_{2}=\frac{\partial f_{w}}{\partial S_{g}}, \\
q_{3}=\frac{\partial f_{g}}{\partial S_{w}}, & q_{4}=\frac{\partial f_{g}}{\partial S_{g}} .
\end{array}
$$



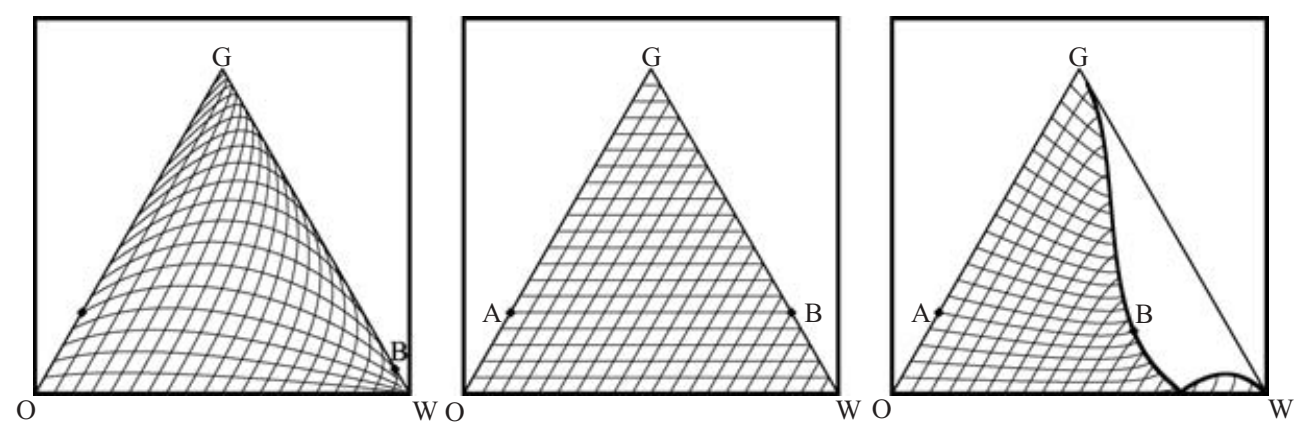

FIGURE 3. Composition paths in the triangular composition diagram, for case $\mathrm{I}, M=1$ and for various values of $\Lambda_{m}$ (equal to $0.2,1$ and 5, respectively). When $\Lambda_{m}<1$, the problem is hyperbolic. When $\Lambda_{m}=1$, the paths are straight lines. When $\Lambda_{m}>1$, a region of ellipticity develops near the GW axis, which increases in size as $\Lambda_{m}$ increases. Point A denotes the injection state for Riemann problem R1.

The eigenvalues of the matrix

$$
\lambda_{ \pm}=\frac{\left(q_{1}+q_{4}\right) \pm \sqrt{\left(q_{1}-q_{4}\right)^{2}+4 q_{2} q_{3}}}{2}
$$

determine the nature of the problem. Two real and distinct eigenvalues define a genuinely hyperbolic system, in which case the solution of a Riemann problem is a combination of rarefaction waves and shocks. The first are the integral solutions of the right eigenvectors of $\boldsymbol{A}$. Solutions corresponding to eigenvalue $\lambda_{+}$(fast or $\lambda_{+}$ paths) are obtained from

$$
\frac{\mathrm{d} S_{w}}{\mathrm{~d} S_{g}}=\frac{q_{2}}{\lambda_{+}-q_{1}},
$$

and solutions corresponding to $\lambda_{-}$(slow or $\lambda_{-}$paths) from

$$
\frac{\mathrm{d} S_{w}}{\mathrm{~d} S_{g}}=\frac{\lambda_{-}-q_{4}}{q_{3}} .
$$

Shocks are obtained from the solution of the corresponding Hugoniot conditions, which for given left and right states (superscripts $l$ and $r$, respectively) read as

$$
v=\frac{f_{w}\left(S_{w}^{r}, S_{g}^{r}\right)-f_{w}\left(S_{w}^{l}, S_{g}^{l}\right)}{S_{w}^{r}-S_{w}^{l}}=\frac{f_{g}\left(S_{w}^{r}, S_{g}^{r}\right)-f_{g}\left(S_{w}^{l}, S_{g}^{l}\right)}{S_{g}^{r}-S_{g}^{l}},
$$

where $v$ is the shock velocity. However, shocks will violate the parallel-flow approximation. YY showed that they may also lead to erroneous results or an undeterminacy, unless additional physics are included, essentially corresponding to non-classical shocks (LeFloch 2002). For this reason, we will concentrate on Riemann problems that give rise to rarefaction waves, except when an elliptic region is to be traversed, which of course is the main interest of this paper.

In the following, we will use a triangular 'composition' diagram to represent the various quantities (figure 3). The quasi-linear formalism allows for the solution of all dependent variables to be expressed as a function of one of them. Hence, solution paths can be traced in a two-dimensional space, which for convenience in the present context we take as a triangular diagram. Axis OG $\left(S_{w}=0\right)$, where $q_{2}=0$, is a $\lambda_{+}$path, 
axis OW $\left(S_{g}=0\right)$, where $q_{3}=0$, is a $\lambda_{-}$path. Along the latter, the two eigenvalues are independent of $M$, and given by the respective expressions

$$
\lambda_{ \pm}=\frac{3}{2} \frac{\left[1+\left(2 \Lambda_{m}-3\right) S_{o}^{2}-2\left(\Lambda_{m}-1\right) S_{o}^{3}\right]}{\left[1+\left(\Lambda_{m}-1\right) S_{o}^{3}\right]^{2}} \text { or } \frac{3}{2} \frac{\left[1+\left(\Lambda_{m}-1\right) S_{o}^{2}\right]}{\left[1+\left(\Lambda_{m}-1\right) S_{o}^{3}\right]},
$$

corresponding to $\lambda_{+}$or to $\lambda_{-}$, depending on which of the two expressions is greater. The first is identical to the velocity given in YY. Of importance are umbilic points and coincidence curves (Keyfitz 1989; Shearer \& Trangenstein 1989) defined as the points or the curves on which the two eigenvalues are equal. Along the various axes, specific umbilic points exist. For example, on the OW axis, the location of the umbilic point is given from the solution of the cubic equation

$$
\left(\Lambda_{m}-1\right)^{2} S_{o}^{3}+3\left(\Lambda_{m}-1\right) S_{o}-\left(\Lambda_{m}-2\right)=0,
$$

for $\Lambda_{m}>2$. Vertices $\mathrm{G}$ and $\mathrm{W}$ are umbilic points. Examples of coincidence curves will be given below. The path corresponding to the largest eigenvalue becomes tangent to the GW axis $\left(S_{o}=0\right)$. Along this axis, the eigenvalues are independent of $\Lambda_{m}$ and equal to

$$
\lambda_{+}=\frac{3}{2} \frac{\left[1+(2 M-3) S_{g}^{2}-2(M-1) S_{g}^{3}\right]}{\left[1+(M-1) S_{g}^{3}\right]^{2}}
$$

and

$$
\lambda_{-}=\frac{3}{2} \frac{\left(1-S_{g}^{2}\right)}{\left[1+(M-1) S_{g}^{3}\right]},
$$

if $M>1$ (and conversely, if $M<1$ ). Expression (25) coincides with the velocity in YY. When $M=1$, both paths become tangent to GW (see below), while the eigenvalues reduce to the Poiseuille velocity $3\left(1-S_{g}^{2}\right) / 2$. The entire axis $\mathrm{GW}$ is then a coincidence curve.

For some combination of parameters, the eigenvalues may become complex $\left(\left(q_{1}-q_{4}\right)^{2}+4 q_{2} q_{3}<0\right)$, in which case the system has a region of elliptic behaviour. It will be shown below that a non-monotonic mobility profile is necessary for the development of an elliptic region and a change of type. For convenience, we will consider three different cases, special limits of which allow for analytical solutions.

\section{Mixed-type behaviour}

\section{Case $I . M=1$ and $\Lambda_{m} \neq 1$}

The case $M=1, \Lambda_{m} \neq 1$ is the simplest model of non-monotonic behaviour in the mobility-concentration function. It also allows for analytical insight. For this we introduce parameter $\epsilon_{1} \equiv \Lambda_{m}-1$, and expand at small $\epsilon_{1}$ to obtain

$$
q_{1}=3 S_{w}-\frac{3}{2} S_{w}^{2}+O\left(\epsilon_{1}\right), \quad q_{2}=O\left(\epsilon_{1}\right), \quad q_{3}=O\left(\epsilon_{1}\right), \quad q_{4}=\frac{3}{2}\left(1-S_{g}^{2}\right)+O\left(\epsilon_{1}\right) .
$$

In the limit $\epsilon_{1}=0\left(\Lambda_{m}=1\right)$, matrix $\boldsymbol{A}$ diagonalizes and the problem reduces to the 'two-fluid' problem of YY. The eigenvalues $\lambda_{+}$and $\lambda_{-}$become sole functions of $S_{w}$ and $S_{g}$, respectively, while the composition paths become straight lines parallel to the $S_{w}$ and $S_{g}$ axes in the composition diagram (figure 3).

When $\epsilon_{1} \neq 0\left(\Lambda_{m} \neq 1\right)$, the eigenvalues become complex in a region, around the axis $\mathrm{GW}$, which expands with increasing $\left|\epsilon_{1}\right|$. At small $\left|\epsilon_{1}\right|$, this region is bounded by the 
axis GW and the curve

$$
S_{o} \equiv U_{1}\left(S_{w}\right)=2\left(\Lambda_{m}-1\right)\left(1-S_{w}\right) S_{w}^{2}\left(3-S_{w}\right) .
$$

Clearly, for the elliptic region to be physically relevant, condition $\Lambda_{m}>1$ must apply (when $\Lambda_{m}<1$, the region of ellipticity lies outside the physically meaningful region, and it is of no physical interest). Physically, this corresponds to the situation of a concave mobility profile, where a more viscous initial fluid is being displaced by an intermediate, less viscous fluid, which is itself being displaced by a more viscous fluid.

We infer that the system is hyperbolic, when the mobility profile across the half-gap is concave downstream, but gives rise to a mixed-type problem when it is convex downstream. We stress that the development of an elliptic region arises here in the absence of any empirical assumptions. It is based solely on the parallel flow (equilibrium) approximation and the non-monotonicity of the mobility profile. This is to be contrasted, for example, to the three-phase flow problem in porous media where elliptic regions have often been attributed to incorrectly modelled empirical relative permeability functions (Juanes \& Patzek 2003). We also note that non-monotonicity in the constitutive equations, e.g. the equation of state in the case of van der Waals fluid dynamics or the stress-strain relation in the case of elastodynamics, is likewise the cause of mixed-type behaviour in the corresponding two problems (Truskinovsky 1987; Shearer \& Yang 1995).

Solution paths and the region of ellipticity in the triangular diagrams are shown in figure 3 for $M=1$ and various values of $\Lambda_{m}$. As mentioned above, elliptic behaviour occurs only if $\Lambda_{m}>1$, and in a region which expands as $\Lambda_{m}$ increases above 1 . The paths become increasingly curved, as $\Lambda_{m}$ deviates from 1 , with a curvature sign which depends on whether or not $\Lambda_{m}$ is less than or greater than 1 . When $\Lambda_{m}<1$, the paths become tangential to the GW axis (at the points where $q_{1}-q_{4}=2 q_{2}=-2 q_{3}$ ). Near the point of tangency $\mathrm{B}$, the equation of the path can be approximated by

$$
S_{w} \approx 1-S_{g}-\frac{1}{2\left(1-\Lambda_{m}\right)}\left[\int_{S_{g}(\mathrm{~B})}^{S_{g}} \frac{\mathrm{d} x}{(1-x) \sqrt{x(2+x)}}\right]^{2} .
$$

When $\Lambda_{m}>1$, the two paths terminate at the boundary of the elliptic region, where their slopes become equal. Noteworthy is the development of a lobe of genuine hyperbolicity near the apex $\mathrm{W}$.

Case II. $M \neq 1$ and $\Lambda_{m} \neq M$

A second generic case corresponds to $M \neq 1$. Analytical results are possible when parameter $\epsilon_{2} \equiv \Lambda_{m}-M$ is small. Now, we have the expansions

$$
\begin{array}{ll}
q_{1}=q_{10}\left(S_{w}\right)+O\left(\epsilon_{2}\right), & q_{2}=3 \epsilon_{2} S_{g}^{2} q_{21}\left(S_{w}\right)+O\left(\epsilon_{2}^{2}\right), \\
q_{3}=q_{30}\left(S_{w}, S_{g}\right)+O\left(\epsilon_{2}\right), & q_{4}=q_{40}\left(S_{w}, S_{g}\right)+O\left(\epsilon_{2}\right),
\end{array}
$$

where the leading-order terms are

$$
\left.q_{10}\left(S_{w}\right)=\frac{3 S_{w}}{2 E^{2}}\left[2-S_{w}+2(M-1)\right)\left(1-S_{w}\right)^{2}\right],
$$

where $E \equiv 1+(M-1)\left(1-S_{w}\right)^{3}$, and

$$
q_{40}\left(S_{w}, S_{g}\right)=\frac{3}{2 E}\left[1+(M-1)\left(1-S_{w}\right)^{2}-M S_{g}^{2}\right] .
$$


In the limit $\epsilon_{2}=0\left(\Lambda_{m}=M\right)$, matrix $\boldsymbol{A}$ becomes lower triangular, as $q_{2}=O(\epsilon) \rightarrow 0$. Then, the eigenvalues are equal to $q_{10}$ or $q_{40}$. This case is another version of the 'two-fluid' problem discussed in YY.

As $\Lambda_{m}$ deviates from $M$, an elliptic region develops around the coincidence curve, given by

$$
S_{g} \equiv U_{2}\left(S_{w}\right)=\left(1-S_{w}\right) \sqrt{1-\frac{S_{w}^{2}\left(3-S_{w}\right)(M-1)}{M\left[1+(M-1)\left(1-S_{w}\right)^{3}\right]}}
$$

provided that $\Lambda_{m}-M>0$, namely that the profile is non-monotonic (and with the restriction $\Lambda_{m}>1$ ). To show this, we expand around the coincidence curve for small $\epsilon_{2}$ and introduce the expansions given in (30) in the condition of the vanishing of the discriminant

$$
\left(q_{1}-q_{4}\right)^{2}=-4 q_{2} q_{3} .
$$

To match the right-hand side of the above, which is $O\left(\epsilon_{2}\right)$, the boundary of the elliptic region must be perturbed in the form

$$
S_{g}=U_{2}\left(S_{w}\right)+\sqrt{\left|\epsilon_{2}\right|} \phi\left(S_{w}\right)+O\left(\epsilon_{2}\right) .
$$

Noting that $S_{g}=U_{2}\left(S_{w}\right)$ is the solution of $q_{10}=q_{40}$, provided that $M>1$, we then obtain the following equation for $\phi$

$$
\left(\left.\phi \frac{\partial q_{40}}{\partial S_{g}}\right|_{S_{g}=U_{2}}\right)^{2}=-12 \operatorname{sgn}\left(\epsilon_{2}\right) q_{21} S_{w}^{2} q_{30}\left(S_{w}, U_{2}\left(S_{w}\right)\right),
$$

where $\operatorname{sgn}\left(\epsilon_{2}\right) \equiv \epsilon_{2} /\left|\epsilon_{2}\right|$. Now, it can be shown that $\left(\Lambda_{m}-1\right) q_{30}\left(S_{w}, U_{2}\left(S_{w}\right)\right)<0$, thus (36) admits a (two-branch) solution, only if

$$
\epsilon_{2}\left(\Lambda_{m}-1\right)=\left(\Lambda_{m}-M\right)\left(\Lambda_{m}-1\right)>0 .
$$

In view of the restriction $\Lambda_{m}-1>0$, condition (37) is again a condition of nonmonotonicity, thus proving the claim made. The region of ellipticity is bounded by the two solutions of (36). Its size increases with increasing $\epsilon_{2}$, but remains within the physically meaningful space (as shown in figure 4). In contrast, if the profile is monotonic, an elliptic region does not develop (figure 4), although a coincidence curve exists. Composition paths and other properties are shown in figure 4.

Case III. $M \neq 1$ and $\Lambda_{m} \neq 1$

The generic case $M \neq 1$ and $\Lambda_{m} \neq 1$, is a third case where analytical results are possible, when parameter $\epsilon_{3}=\Lambda_{m}-1$ is small. The approach is analogous to the previous and for simplicity will not be repeated. Details can be found in Shariati \& Yortsos (2001). As before, a non-monotonic profile is required for the existence of an elliptic region.

\section{The solution of Riemann problems}

We will proceed next with the solution of two illustrative Riemann problems, one with injection at a point $\mathrm{A}$ along the $\mathrm{OG}$ axis and initial condition at $\mathrm{W}$ (problem R1) and another with injection at $\mathrm{G}$ and initial condition at $\mathrm{W}$ (problem R2). Emphasis will be placed only on case I, which is representative of the other two cases as well. We will develop analytical results, when possible, and compare them with numerical solutions of a diffusion-augmented model, and, in a subsequent 

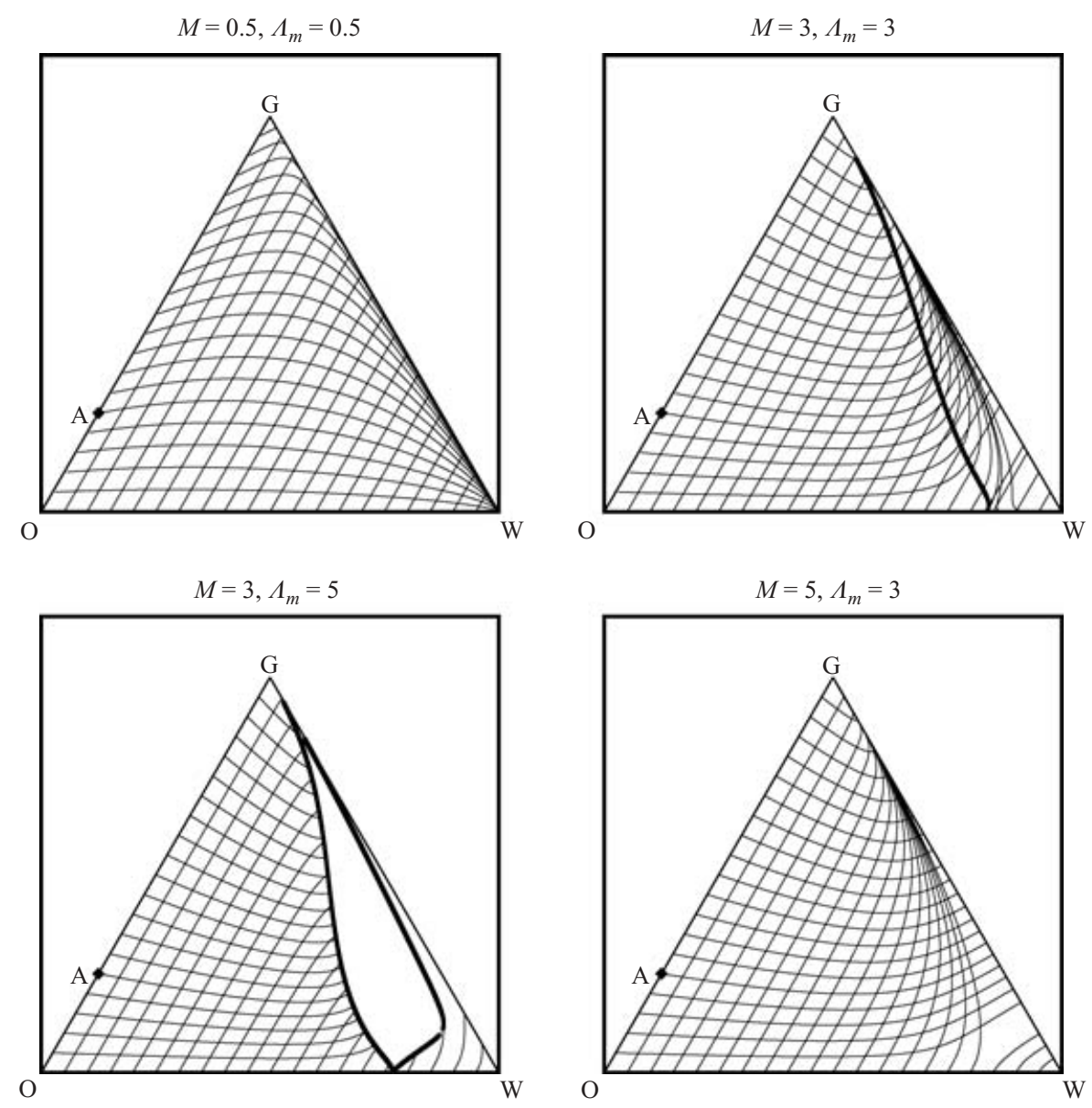

FIGURE 4. Composition paths in the triangular composition diagram, for case II, and for various values of $M$ and $\Lambda_{m}$ (equal to $(0.5,0.5),(3,3),(3,5)$ and $(5,3)$, respectively). When $\Lambda_{m}=M>1$, a coincidence curve exists joining apex $\mathrm{G}$ to the axis $\mathrm{OW}$. An elliptic region develops if $\Lambda_{m}>M>2$. Point A denotes the injection state for Riemann problem R1.

section, with the numerical solution of the full, higher-dimensional model. As pointed out, we have considered Riemann problems such that in the hyperbolic region their solution is given only by rarefaction waves. Riemann problems can easily be realized experimentally, by injecting the fluids symmetrically across the gap at fractional flow values corresponding to the desired values of the inlet volume fractions, as determined from expressions (14) and (15).

Analytical results are possible in the case of genuine hyperbolicity, or in the hyperbolic region of the mixed-type case. Adding diffusion to the model requires a numerical solution, which can be obtained in two ways: (i) by an ad hoc addition of diffusion on the right-hand side of the hyperbolic equations (16); (ii) by retaining the diffusivity $N_{T D}$ in the original system (6)-(9), always subject to the parallel-flow approximation. Because of its better physical basis, we proceeded using the second approach.

The corresponding numerical problem can be solved by a variety of methods, e.g. standard finite-difference schemes as in YY (see also Shariati \& Yortsos 2001). In this paper, we elected to use lattice BGK (LBGK) simulations, however. As we noted above, this lattice gas-related method was successfully used in Rakotomalala et al. 
(1997) to solve the full displacement problem in the monotonic case (the 'two-fluid' problem). Having access to this numerical tool, we also used it to provide numerical solutions to the problem in the presence of diffusion, but under the parallel-flow approximation, which is the subject of interest of this section. For this purpose, the convection-diffusion equation for the concentration was not coupled with the NavierStokes or Stokes equations, but with the 'lubrication' velocity field. The velocity component in the mean flow direction is given by the parallel flow expressions (8)-(9) and the transverse one is obtained from the continuity equation. In all simulations, the following (dimensionless) viscosity dependence was taken

$$
\mu(c)= \begin{cases}M^{-1}+\frac{1}{2}\left(\Lambda_{m}^{-1}-M^{-1}\right) \mathrm{e}^{(c-0.25) / 0.01} & \text { if } c>0.75, \\ \Lambda_{m}^{-1}+\frac{1}{2}\left(M^{-1}-\Lambda_{m}^{-1}\right) \mathrm{e}^{-(c-0.25) / 0.01}+\frac{1}{2}\left(\Lambda_{m}^{-1}-1\right) \mathrm{e}^{(c-0.75) / 0.01} & \text { if } 0.25<c<0.75, \\ 1+\frac{1}{2}\left(\Lambda_{m}^{-1}-1\right) \mathrm{e}^{-(c-0.75) / 0.01} & \text { if } c<0.25 .\end{cases}
$$

The step-like dependence is necessary for the clear identification of the three fluid regions in the present kinematic description.

\subsection{Analytical and numerical results}

Consider, first, the solution of Riemann problem R1, with state A given by $S_{o}=0.75$, $S_{g}=0.25$. Figure 5 shows analytical results for the genuinely hyperbolic case. Plotted are the solution path in the triangular diagram, the saturations, the fluid boundaries, and the transversely averaged concentration, defined as $\bar{c}=S_{g}+S_{o} / 2$, as functions of the similarity variable $x / t$. Because the problem is genuinely hyperbolic and the slow eigenvalue increases monotonically in the direction from injection to initial, the solution lies on the $\lambda_{-}$path $\mathrm{AB}$. This describes first an increasing and then a decreasing value of $S_{g}$, the finger of the fluid in the centre swelling before it sharpens at the tip. The finger of the in-between fluid continuously decreases and near the tip reduces to a thin layer of infinitesimal thickness (in this case of zero diffusion) and reduced mobility. The path encounters the $\mathrm{GW}$ axis at point $\mathrm{B}$, at which it becomes tangential to it, as pointed out above. From then on, the path stays on the GW axis, where the two eigenvalues are the same. Along this part, the solution for $S_{g}$ and $\bar{c}$ is merely the Poiseuille profile.

Thus, in the genuinely hyperbolic case the overall solution is a rarefaction, the leading part being the Poiseuille profile with a tip velocity equal to $3 / 2$. These features are the same as those of the 'two-fluid' problem of YY in the case $M<3 / 2$.

Corresponding results from the simulation of the hyperbolic system are shown in figure 6. The comparison between analytical and numerical results is generally good. Diffusion in the numerical simulation does spread the rarefaction wave, and causes the path in the composition space to shift to the left of the W vertex, on the OW axis, as the layer in-between the two fluids has now a non-zero thickness. Thus, the initial state is not reached tangentially from the WG axis, as the analytical solution requires, but rather from the $\mathrm{OW}$ axis. This mismatch increases progressively with time as $t^{1 / 2}$, and is enhanced with increasing diffusion. The small deviation at the tip reflects a numerical artefact in the numerical simulation, as a result of a slightly different initial condition used in the simulation, when the parallel flow is imposed.

Analytical results for the same problem, but in the case $\Lambda_{m}>1$, where a mixedtype behaviour arises, are shown in figure $7\left(\Lambda_{m}=10\right)$. The analytical construction follows the $\lambda_{-}$path $\mathrm{AE}$, and it is a rarefaction wave, until the path approaches the elliptic region at point $\mathrm{E}$. The path has a curvature opposite to the previous, with $S_{g}$ 

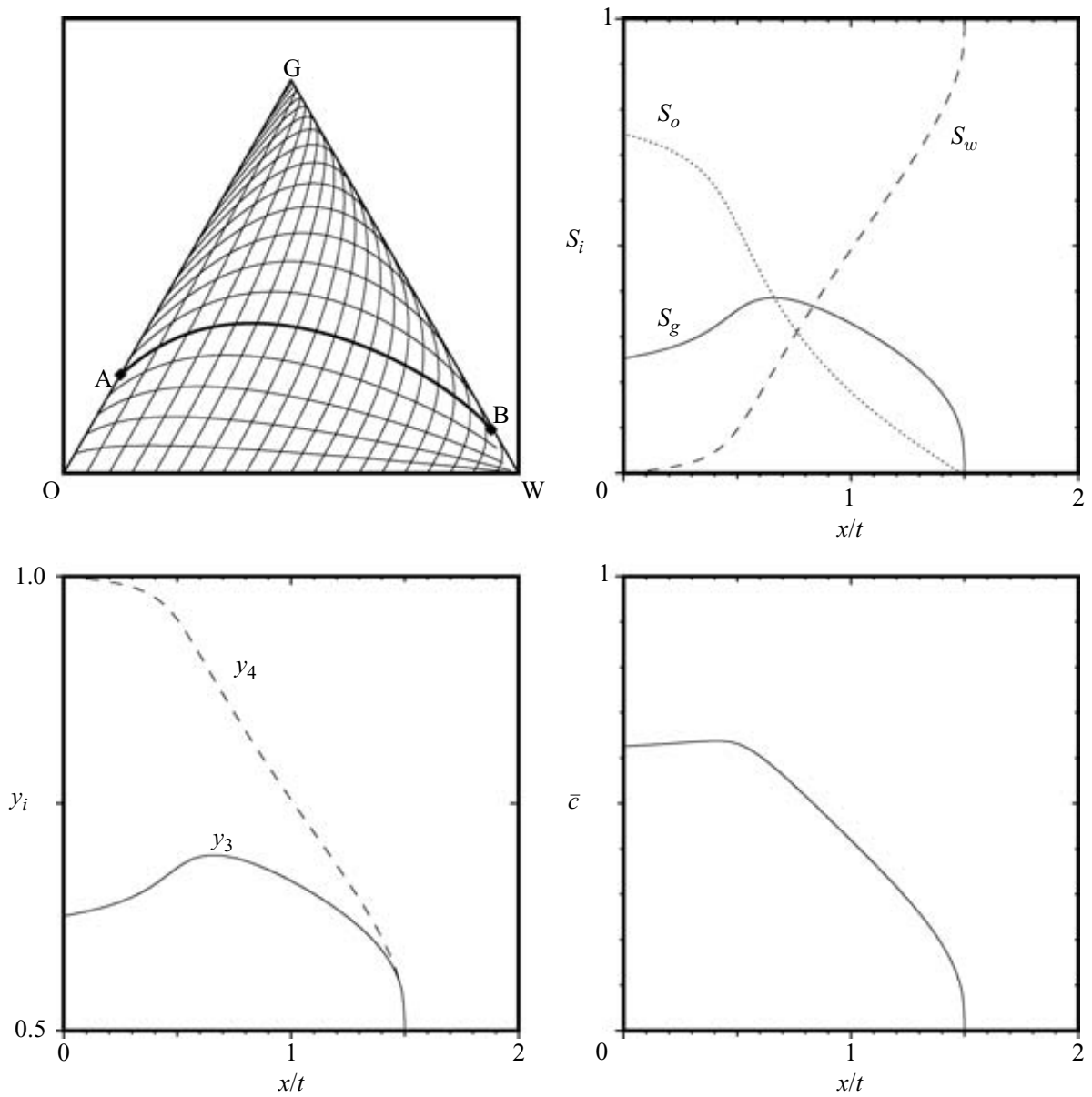

FIGURE 5. The analytical solution of Riemann problem R1 (injection at state A, initial condition at state $\mathrm{W}$ ), for case $\mathrm{I}, M=1$ and for $\Lambda_{m}=0.1$, corresponding to a rarefaction. Clockwise from top left are composition paths, saturation profiles, transversely averaged concentration and phase (finger) boundaries, plotted as functions of the convective similarity variable $x / t$. The solid, dotted and dashed curves correspond to the saturation (volume fraction) of fluids $g, o$ and $w$, respectively.

decreasing for the most part. Now, swelling at the tip occurs inside the elliptic region, as is shown in more detail by the full solution below. Connection to the initial state requires the crossing of the elliptic region with a shock. For further progress, we used LBGK simulations.

Figure 8 shows results from the simulations, under the assumption of parallel flow and using small diffusion, as explained above. There is good agreement with the analytical solution in the hyperbolic region. However, when the solution enters the elliptic region it becomes unstable, leading to sustained oscillations of a bounded variation. Figure 9 shows consecutive snapshots of the corresponding concentration field, where the unphysical instability at the tip is clear (again, the very small needlelike feature at the tip is a numerical artefact due to a slightly modified initial condition). The instabilities observed in figures 8 and 9 are consistent with the instability of the uniform states in the elliptic regions (Batchelor \& Janse van Rensburg 

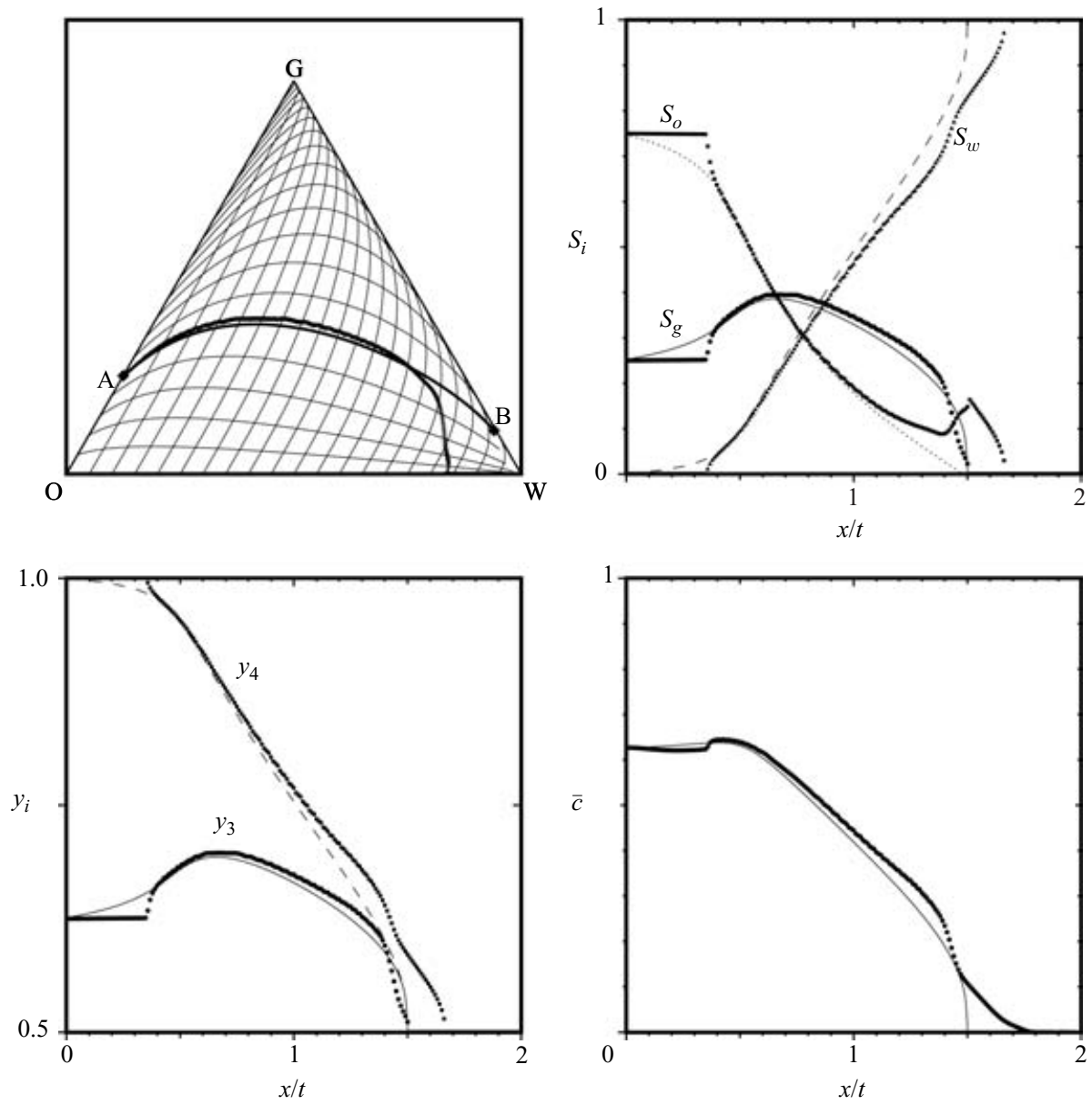

FIgURE 6. The numerical solution of Riemann problem R1 based on the parallel flow approximation for the conditions corresponding to figure 5, with $N_{T D} \epsilon^{2}=2.4 \times 10^{-4}$. Bold curves correspond to the lattice BGK simulation results, all others to the analytical solution. Clockwise from top left are composition paths, saturation profiles, transversely averaged concentration and phase (finger) boundaries, plotted as functions of the convective similarity variable $x / t$. The small deviation from the analytical solution at the tip reflects a numerical artefact in the numerical simulation, as a result of a slightly different initial condition.

1985; Bell et al. 1986; Keyfitz 1989). Diffusion can be stabilizing if it is sufficiently strong to divert the paths from the elliptic region. This is not the case in figures 8 and 9, where the terminal point of the 'three-fluid' region on the OW axis can only be reached through the elliptic region. Figure 10 shows corresponding simulation results under the same conditions, but with increased diffusion. Now, the solution consists of a combination of a 'three-fluid' regime described by the above analytical formalism, with a faster moving 'two-fluid' regime along the OW axis, downstream. The stronger diffusion causes significant departure from the analytical predictions. The transition between the two regimes occurs through a rarefaction wave along the path corresponding to the fast eigenvalue. Because the downstream region is essentially a displacement of a more viscous by a less viscous fluid, however, it leads to a shocklike (finger-like) structure similar to that in YY for the case $M>3 / 2$. We stress that 

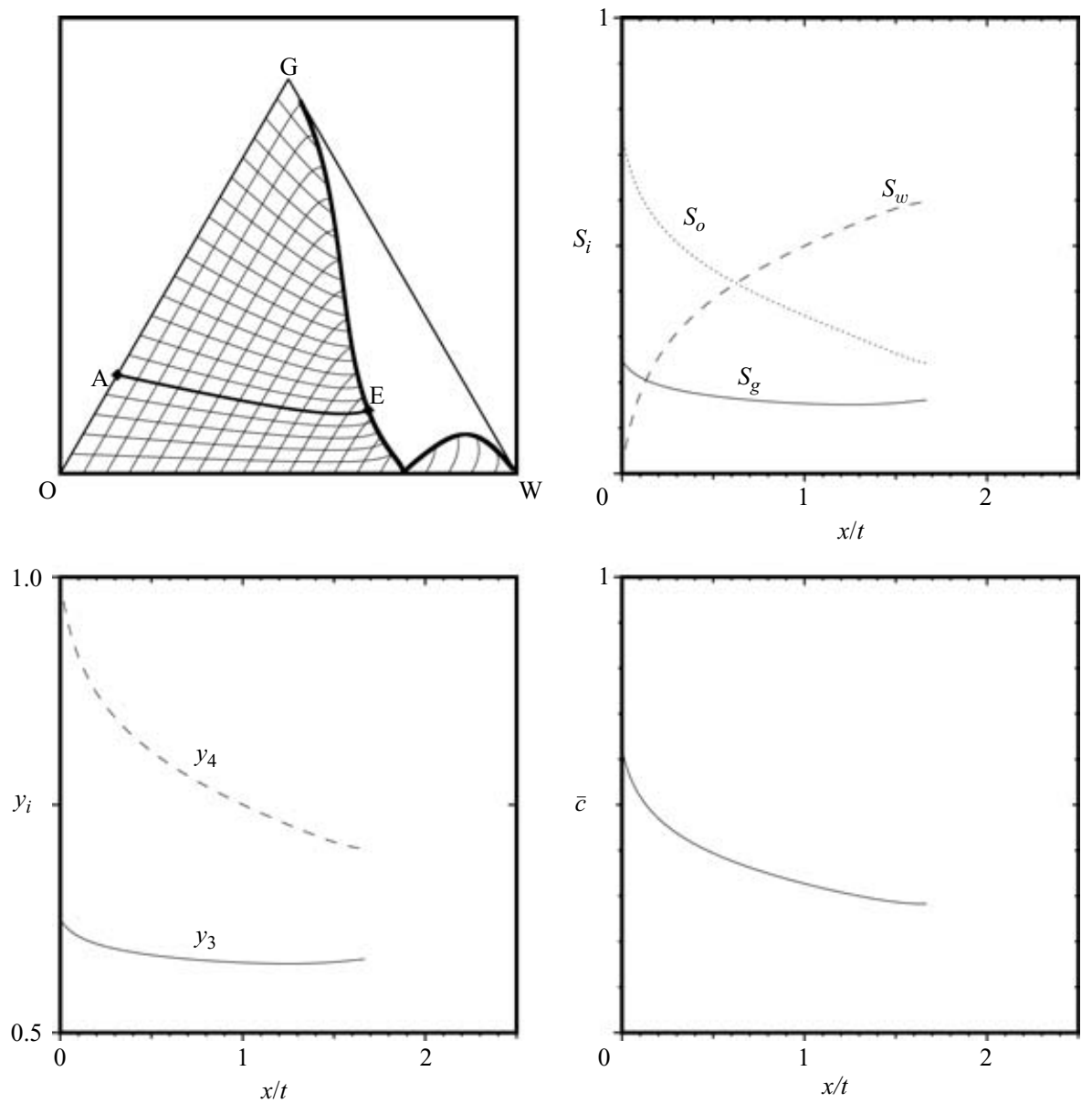

FIGURE 7. The analytical solution of Riemann problem R1 (injection at state A, initial condition at state $\mathrm{W}$ ), for case $\mathrm{I}, M=1$ and for $\Lambda_{m}=10$, corresponding to the case with change-of-type. Clockwise from top left are composition paths, saturation profiles, transversely averaged concentration and phase (finger) boundaries, plotted as functions of the convective similarity variable $x / t$. The rarefaction wave terminates when the elliptic boundary is encountered.

even though this is a mathematically acceptable solution, it is physically questionable, given that across such a shock the parallel-flow assumption, on which this solution is based, will fail, very much as in YY.

We conclude that in the case of change-of-type behaviour, the hyperbolic analysis can correctly capture the features of the displacement, provided that initial and boundary conditions are such that the elliptic region is not crossed, and that the solution in the hyperbolic region is a rarefaction. When the former is not possible, the addition of a small amount of diffusion is not sufficient to prevent unstable behaviour, which will set inside the elliptic region. When shocks develop, they appear to be non-classical. The addition of diffusion simply allows us to bypass the elliptic region but it does not address the issue of crossing it. The following section will address this issue.

Before we proceed, we will use the insight obtained from the R1 problem to extrapolate to the more conventional $\mathrm{R} 2$ problem (injection at $\mathrm{G}$, initial at $\mathrm{W}$ ). In 

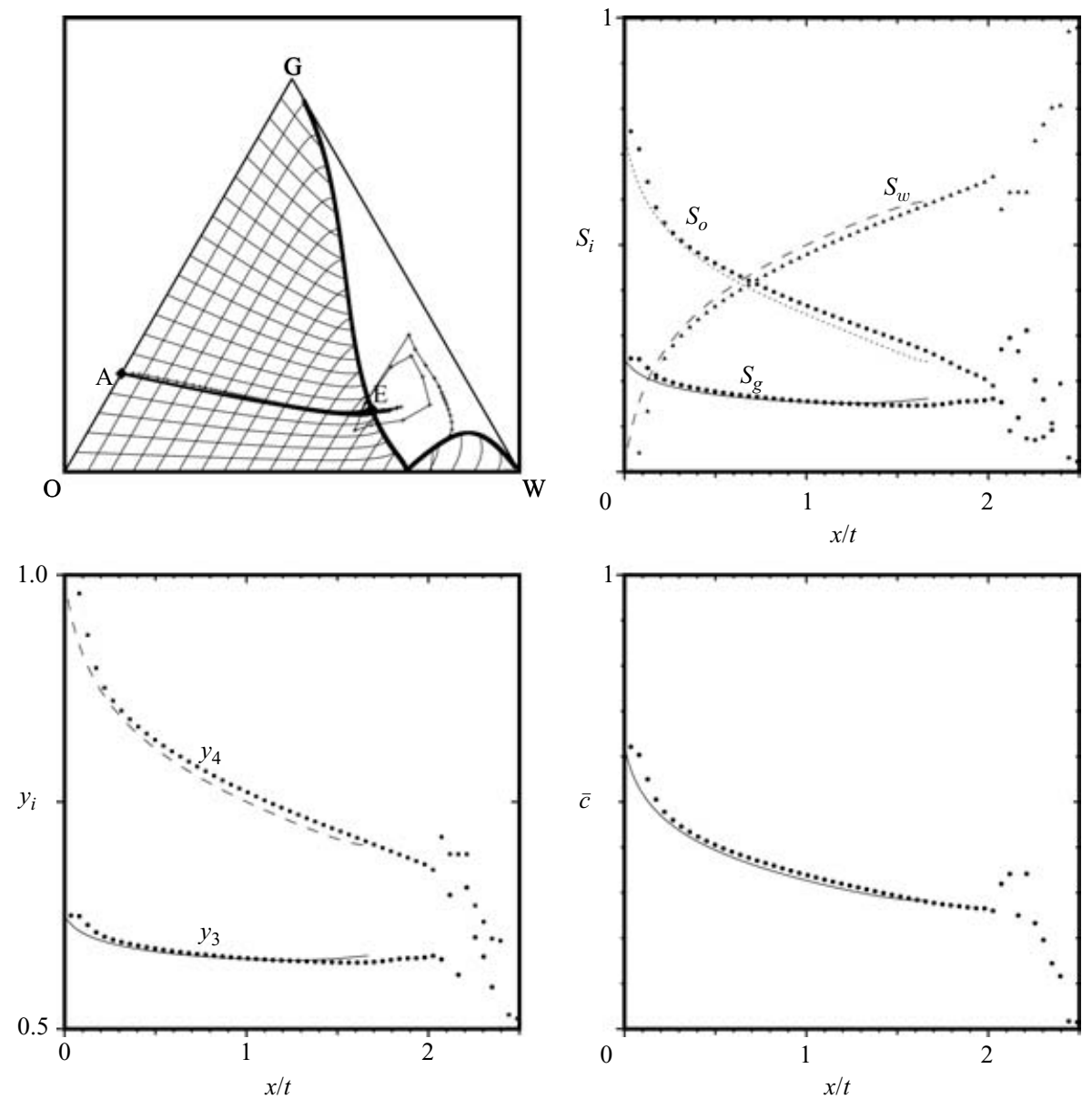

FIGURE 8. The numerical solution of Riemann problem R1 based on the parallel-flow approximation for the conditions corresponding to figure 7, and with a small amount of diffusion added, $N_{T D} \epsilon^{2}=2.4 \times 10^{-4}$. Bold curves correspond to the numerical simulations, all others to the analytical solution. Clockwise from top left are composition paths, saturation profiles, transversely averaged concentration and phase (finger) boundaries, plotted as functions of the convective similarity variable $x / t$. At this level of diffusion, the solution path enters the elliptic region, leading to unstable, oscillatory behaviour. However, the leading part of the solution is a shock, around which the parallel-flow approximation fails, thus invalidating the physical relevance of this solution.

theory, the solution path of the problem in the complete absence of diffusion should be simply the GW axis, namely the Poiseuille profile, as in the case of passive tracer injection. However, the presence of even a small amount of diffusion will introduce a layer of different mobility in-between the two equal mobility fluids. Results from numerical simulations for the hyperbolic case are shown in figure 11. Because of diffusion, the injection state moves from apex $G$ to a point on the $O G$ axis, while the initial state moves from the $\mathrm{W}$ apex to a point to its left on the OW axis. The solution path is a combination of rarefaction waves, along composition paths augmented by diffusion, but otherwise stays close to the Poiseuille profile. Figure 12 shows results corresponding to the mixed-type case. As in the corresponding R1 problem, the numerical solution is based on the parallel-flow approximation with 

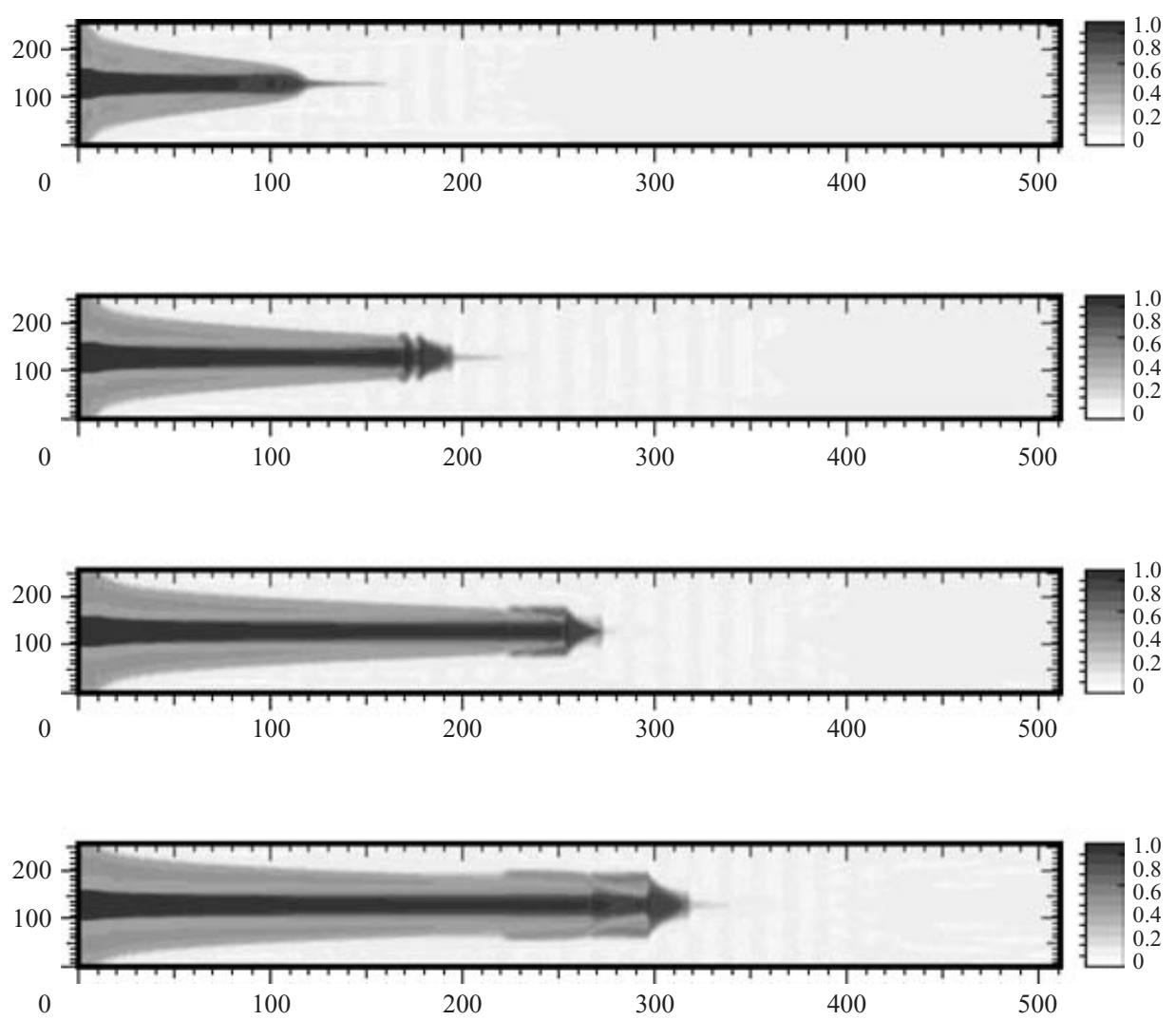

FIGURE 9. Consecutive snapshots of the concentration field across the gap, plotted as functions of the distance $x$, corresponding to the conditions of figure 8 and obtained from the numerical simulations. The region near the tip, where elliptic behaviour develops, shows unstable, oscillatory and unphysical behaviour. The small feature ahead of the front is an artefact of the simulation owing to the use of a modified initial condition.

small diffusion added. We note that as in Riemann problem R1, when the solution paths enter the elliptic region, they become subject to an instability, resulting into unphysical oscillations.

Similar considerations apply for cases II and III discussed above.

\subsection{Crossing the elliptic region}

The above suggest that the only alternative to understanding the displacement when crossing the elliptic region (and more generally when a shock is predicted) is to conduct simulations of the full problem. Indeed, the breakdown of the parallel-flow approximation in these regions signals that additional physics must be inserted. We noted that in the corresponding problems of van der Waals gas dynamics and elastodynamics, this is done by the addition of an effective capillary term, which introduces a third-order derivative to the right-hand side of (1) (e.g. see LeFloch 2002). Here, it will be done exactly, by considering the full problem and using LBGK simulations.

Comparison between analytical and numerical solutions of the full problem for the genuinely hyperbolic case is shown in figure 13. Plotted are the solution paths in the triangular composition diagram, the saturation profiles $\left(S_{g}\right.$ and $\left.S_{o}\right)$ and the fluid 

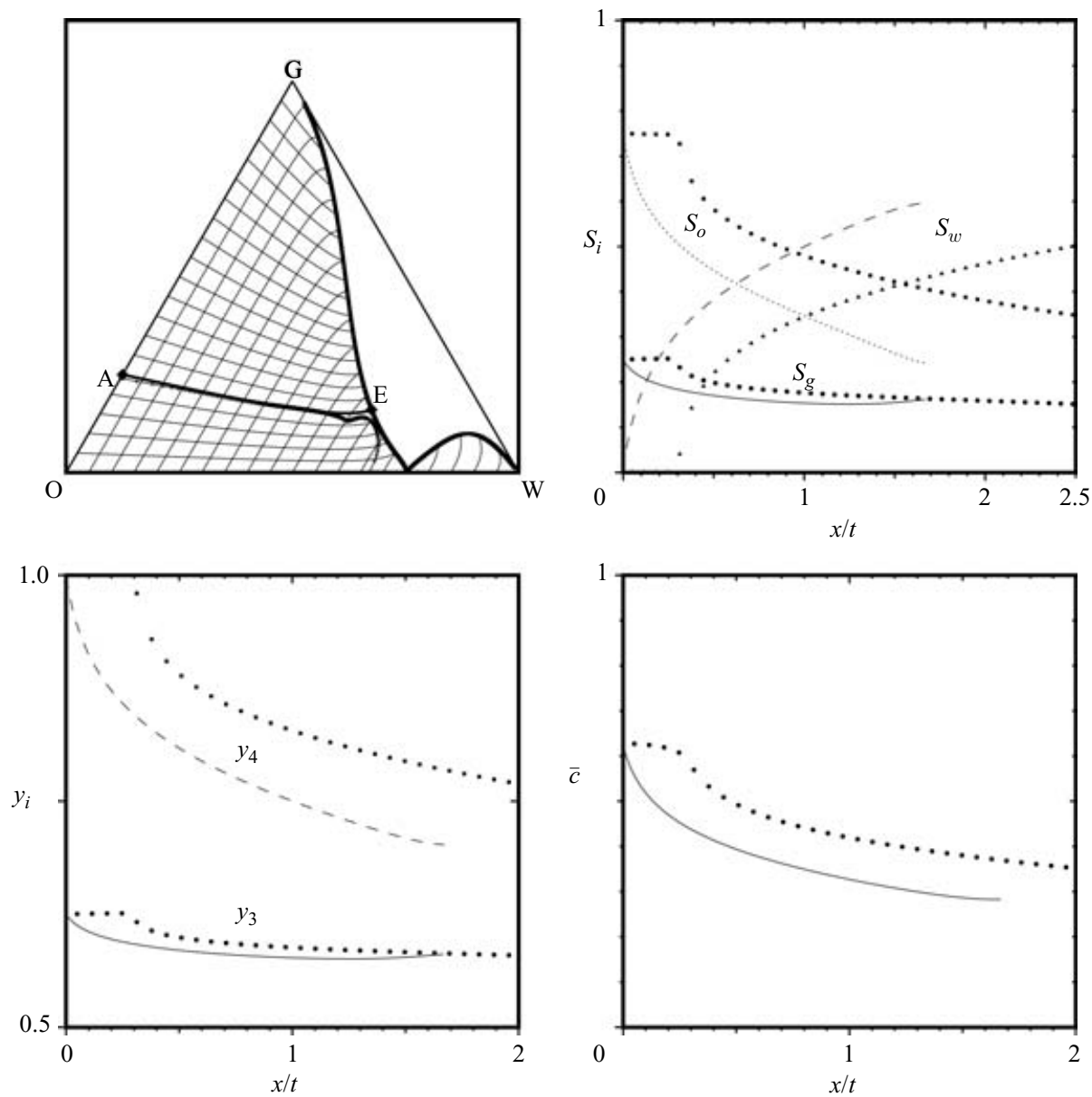

FIGURE 10. The numerical solution of Riemann problem R1 based on the parallel-flow approximation for the conditions corresponding to figure 7, and with a larger amount of diffusion added, $N_{T D} \epsilon^{2}=1.2 \times 10^{-3}$. Bold curves correspond to the numerical simulations, all others to the analytical solution. Clockwise from top left are composition paths, saturation profiles, transversely averaged concentration and phase (finger) boundaries, plotted as functions of the convective similarity variable $x / t$. At this level of diffusion, the solution path does not enter the elliptic region.

boundaries as functions of the similarity variable $x / t$. Because the problem is genuinely hyperbolic and the slow eigenvalue increases monotonically in the direction from injection to initial, the analytical solution lies on the $\lambda_{-}$path $\mathrm{AB}$, as noted previously. Comparison of analytical and simulation results shows a generally good agreement, except near the tip, where a more elaborate structure appears in the numerical solution. Nonetheless, the velocity of the tip is predicted well and it is close to the maximum Poiseuille velocity of $3 / 2$. The presence of diffusion in the numerical model results in the spreading of the rarefaction wave, as before. Thus, although the analytical solution is a sole function of the similarity variable $x / t$, the numerical solution is additionally a function of time. We must note that several transients exist before the asymptotic state of figure 5 is reached. In fact, the asymptotic state is an intermediate one, as at long times diffusion ultimately breaks the convective similarity scaling. Also because of diffusion, the path in the composition space shifts, and the layer of the 

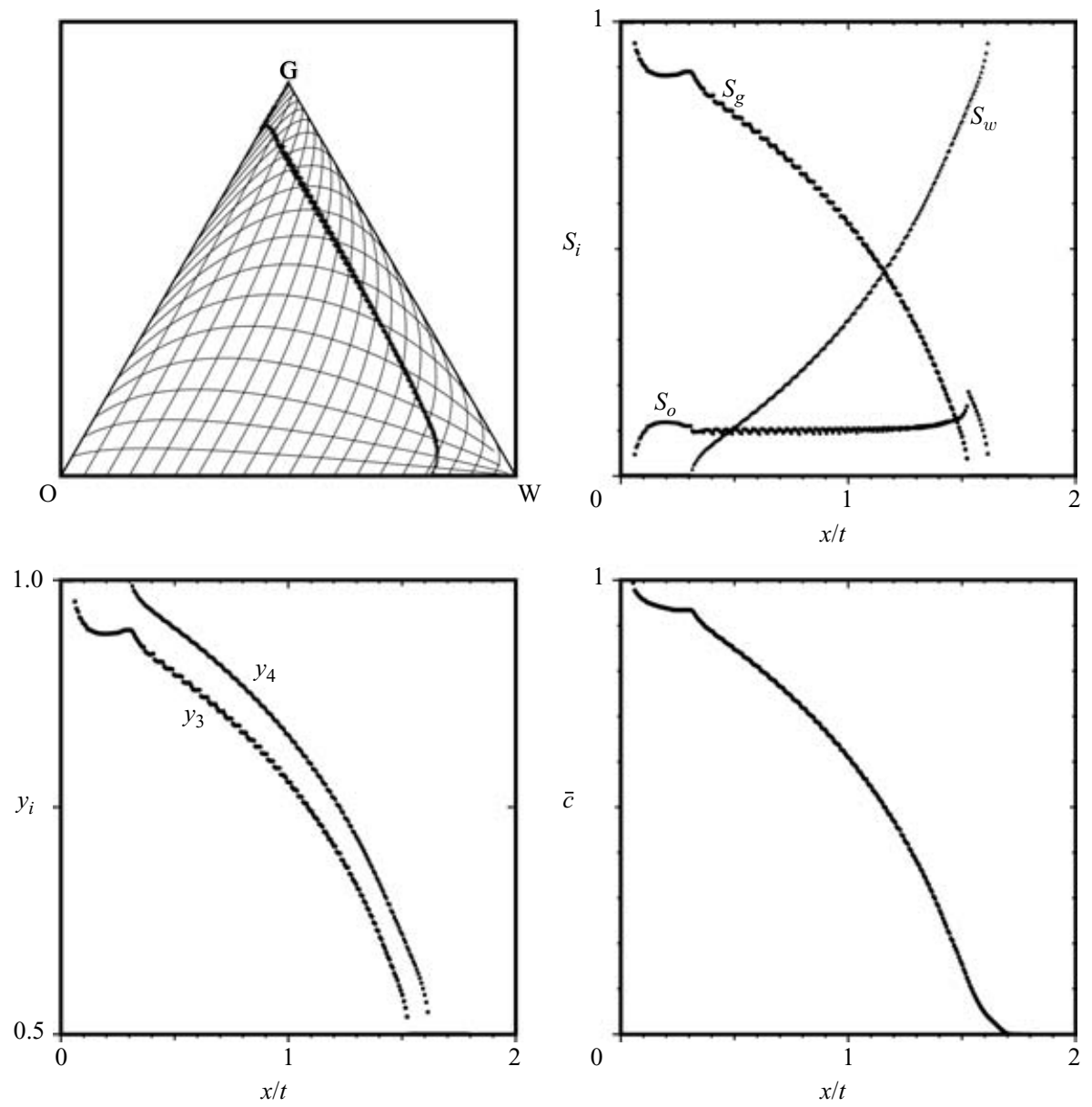

FIGURE 11. The numerical solution of Riemann problem R2 based on the parallel-flow approximation for the conditions corresponding to figure 5, with $N_{T D} \epsilon^{2}=3.1 \times 10^{-5}$. Clockwise from top left are composition paths, saturation profiles, transversely averaged concentration and phase (finger) boundaries, plotted as functions of the convective similarity variable $x / t$. Because of diffusion, the injection state moves from the apex $G$ to a point on the $O G$ axis, while the initial state moves from the $\mathrm{W}$ apex to a point to its left, on the OW axis. The solution is a combination of rarefaction waves along composition paths, but it is close to the Poiseuille profile.

in-between fluid has now a non-zero thickness (as in figure 6). Overall, however, the agreement between numerical and analytical solutions is quite good, showing that the hyperbolic formalism and the parallel-flow assumption are valid approaches in this case.

Figure 14 shows the comparison for the mixed-type case. The numerical results in the figure correspond to an intermediate asymptotic state that is reached after some transients have stabilized. The transient development is shown in the four consecutive snapshots of figures 15-17, where the concentration field vs. the actual distance (figure 15), the paths in the triangular diagram (figure 16), and the saturation profiles plotted vs. the similarity variable (figure 17) are shown. The following features are noted, and were also observed in other simulations with different parameters: 

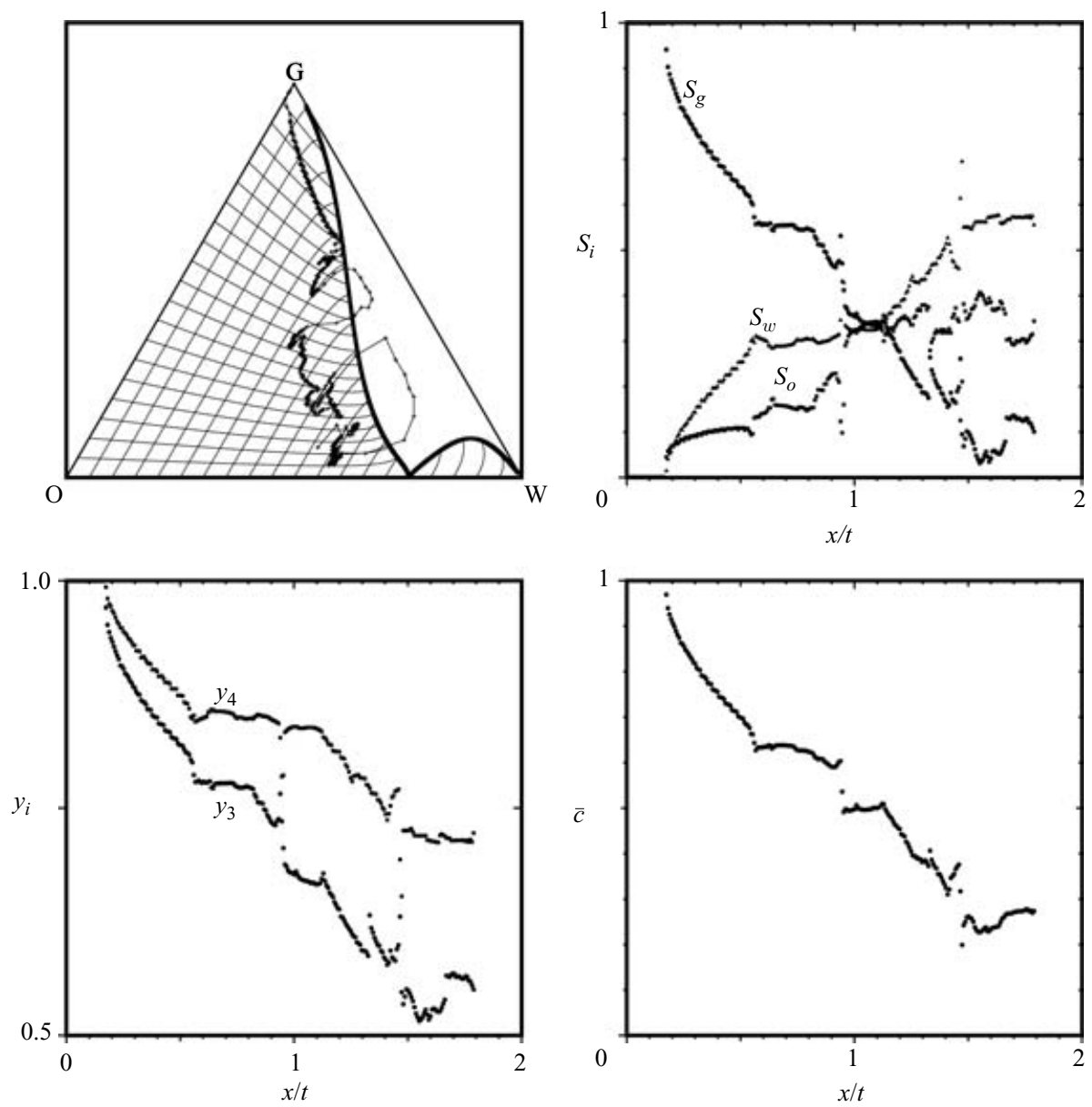

FIGURE 12. The numerical solution of Riemann problem R2 based on the parallel-flow approximation for the conditions corresponding to figure 7, with $N_{T D} \epsilon^{2}=2.4 \times 10^{-4}$. Clockwise from top left are composition paths, saturation profiles, transversely averaged concentration and phase (finger) boundaries, plotted as functions of the convective similarity variable $x / t$. At this level of diffusion, the solution path enters the elliptic region and leads to unstable, oscillatory and unphysical behaviour.

(i) Owing to diffusion, the initial condition rapidly moves to the left of the apex W to point $\mathrm{F}$ (figure 14). This point corresponds to about $10 \%$ volume fraction of fluid $o$ and remains fixed throughout the process. It is the leading edge of the front and it moves with a velocity slightly larger than the Poiseuille velocity of $3 / 2$. The velocity appears to be consistent with the shock velocity that would have been obtained in the two-fluid problem with $M=10$, and states F and $\mathrm{W}$, respectively. That velocity is also slightly larger than the Poiseuille maximum.

(ii) Upstream, the trajectory follows roughly the solution path FE of an almost constant velocity (figure 14), where the volume fraction of fluid $o$ decreases to an almost constant value, at point $\mathrm{E}$. The velocity is approximately the same as the slowest eigenvalue in the lower lobe where the hyperbolic formalism applies. It appears as if points $\mathrm{E}$ and $\mathrm{F}$ were selected such that the velocity on $\mathrm{FE}$ is the same as the shock velocity FW. If so, that would be consistent with a quasi-linear hyperbolic formalism. 

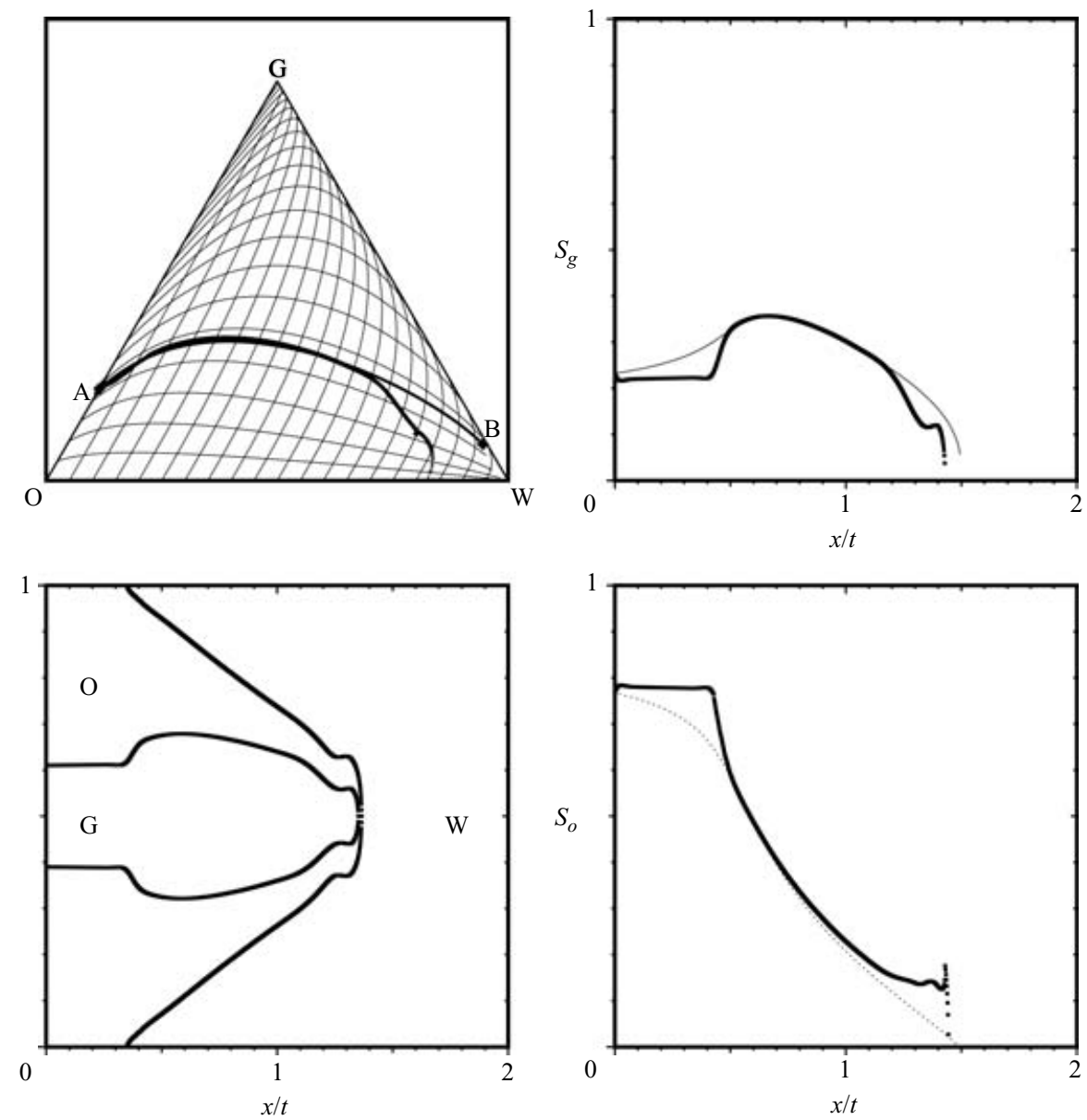

FIGURE 13. Solution of Riemann problem R1 for the conditions of figure $5\left(M=1, \Lambda_{m}=0.1\right.$, genuinely hyperbolic case, with small diffusion, $N_{T D} \epsilon^{2}=1.5 \times 10^{-5}$ ). Comparison between analytical and numerical solutions using the full Navier-Stokes equation. Bold lines correspond to the numerical solutions. Clockwise from top left are composition paths, saturation profiles $S_{g}$ and $S_{o}$ and phase (finger) boundaries, plotted as functions of the convective similarity variable $x / t$.

(iii) The system then locks into a path ED inside the elliptic region. Along this path, the volume fraction of fluid $o$ remains constant, but that of fluid $g$ increases in the upstream direction until a maximum value is reached. During this process, the finger of the latter fluid swells, the swelling increasing until the state is reached. Stages 2 and 3 are at the leading edge of the front, where cross-flow becomes important. None of the features in these regions is predictable with the quasi-linear formalism.

(iv) State $\mathrm{D}$ is connected upstream with point $\mathrm{B}$, outside the elliptic region, apparently through a shock, moving along paths of the fast eigenvalue with a shock velocity consistent with the Rankine-Hugoniot conditions. Along this path, the volume fraction of fluid $g$ decreases upstream, while that of fluid $o$ increases.

(v) Finally, state B is connected further upstream to the injection state. The path follows nicely the quasi-linear formalism. In the development of this state, there appears to be a reversal along the hyperbolic path AB. According to the classical 

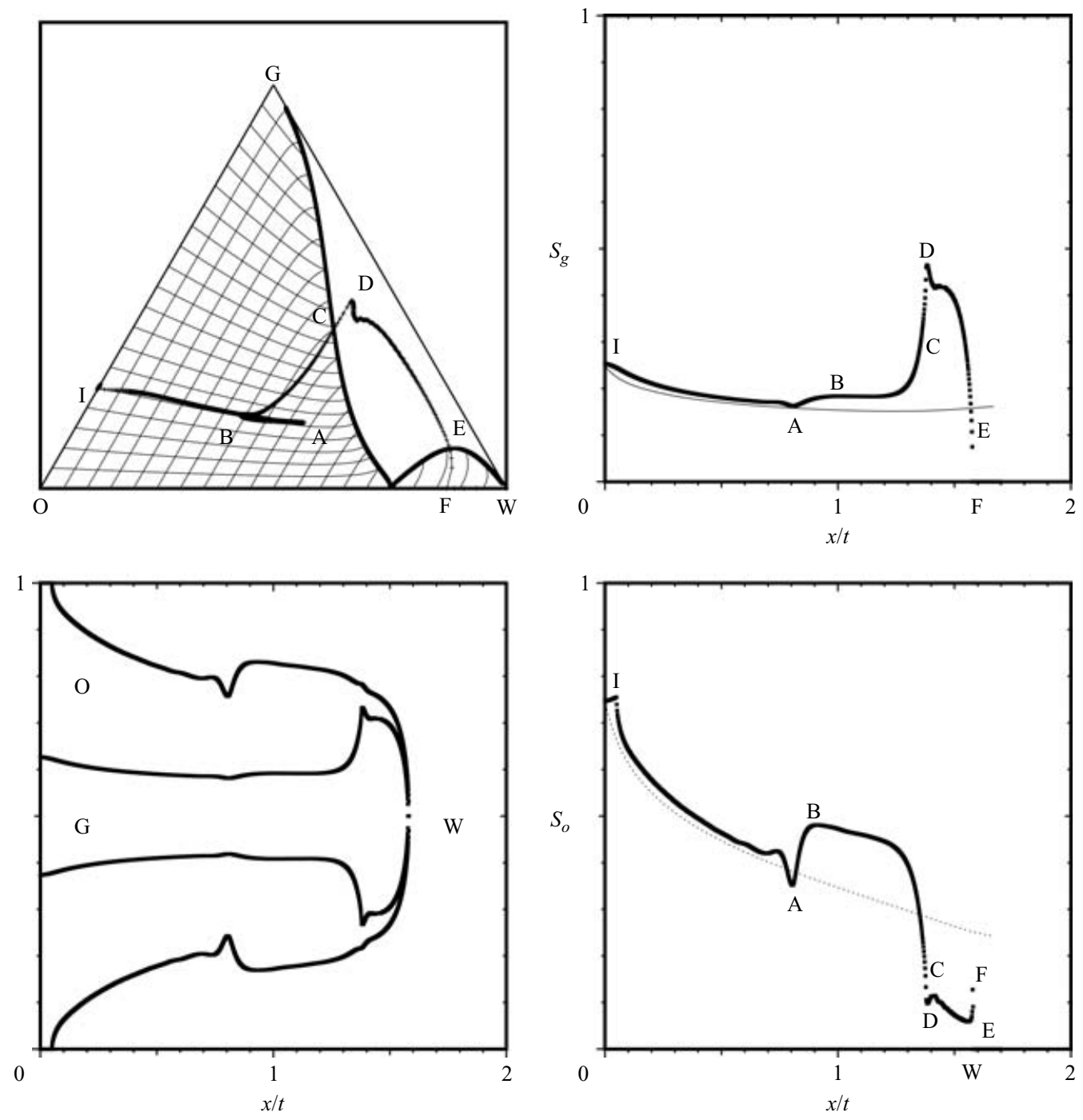

FigURE 14. Solution of Riemann problem R1 for the conditions of figure $7\left(M=1, \Lambda_{m}=10\right.$, mixed-type behaviour, with small diffusion, $\left.N_{T D} \epsilon^{2}=1.5 \times 10^{-5}\right)$. Comparison between analytical and numerical solutions using the full Navier-Stokes equation. Bold lines correspond to the numerical solutions. Clockwise from top left are triangular composition paths, gas saturation profiles and oil and a snapshot of the numerical profiles across the gap, plotted as functions of the convective similarity variable $x / t$.

theory, such a reversal should be accompanied by a shock, which must travel with a velocity smaller than that of point $\mathrm{A}$. We suspect, however, that this feature is associated with weak inertia, which exists in the LBGK simulations, and the resulting instability at small Reynolds numbers.

The behaviour of the full solution as the elliptic region is crossed cannot be captured by the simpler quasi-linear hyperbolic system, even if a small amount of diffusion were to be added. As the simulations show, the elliptic region develops precisely at the tip of the displacement, where the variables undergo sharp changes, and where the parallelflow assumption itself breaks down. The discrepancy in the velocity component in 

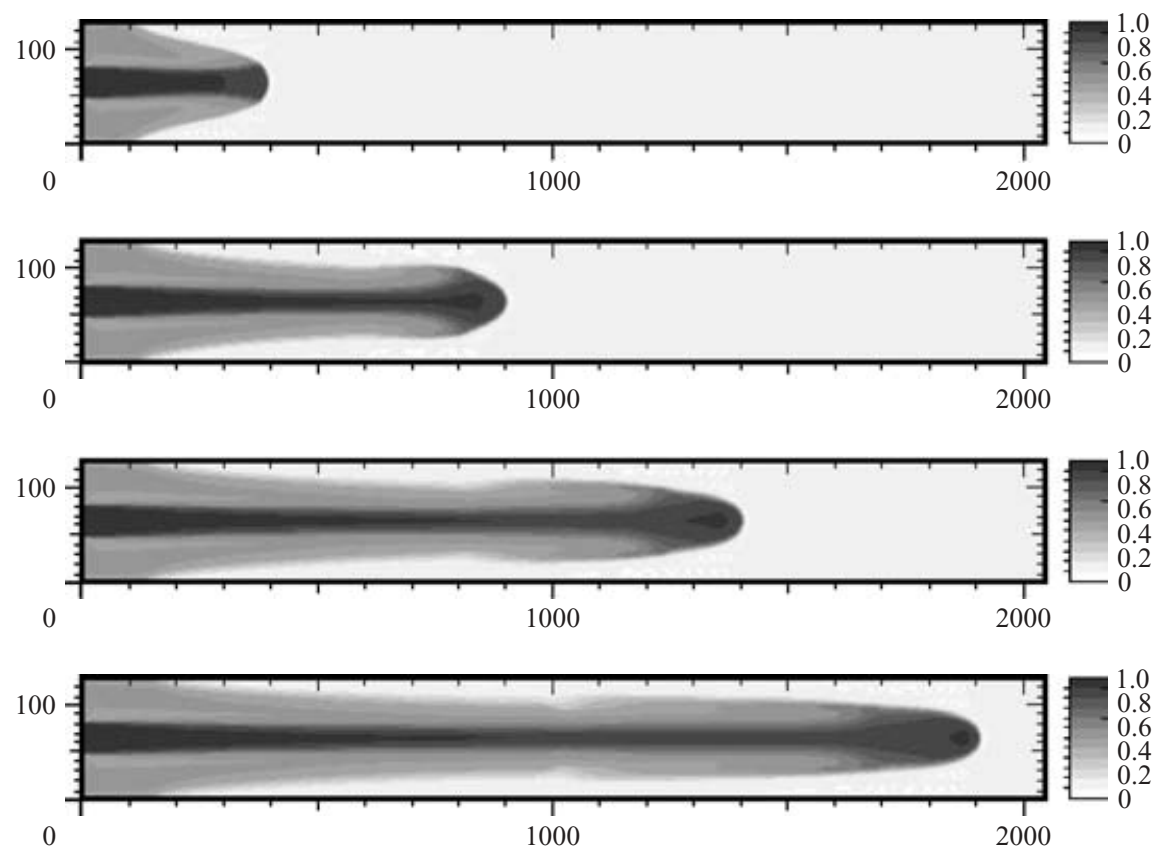

FIgURE 15. Consecutive snapshots of the concentration field across the gap, plotted as functions of the distance $x$, corresponding to the conditions of figure 14 and obtained from the numerical simulations.

the direction of the displacement between the full simulation and the parallel flow expressions (8)-(9) is shown in figure 18. Considerable differences exist near the tip (and also near the end points in the simulation domain). Just as in YY, the quasilinear hyperbolic formalism can reproduce well the features of the 'three-fluid' problem upstream of the tip. However, also as in YY, it fails to capture the tip structure and the velocity of the shock, the resolution of which requires that more detailed physics are included in the description. We are led to conclude, therefore, that the emergence of a mixed-type region is a warning of the local failure of the parallel-flow approximation, which was fundamental for the development of the quasi-linear hyperbolic formalism. Resolving the problem that arises cannot be done simply by using different fractional flow expressions, which will lead to a genuine hyperbolicity, but rather necessitates the solution of the full higher-dimensional problem in that region. In the analogous problems in the dynamics of real fluids, where similar elliptic behaviour is encountered, the resolution in the elliptic region is obtained by introducing an effective capillary term, which results into a nonlinear eigenvalue problem for the determination of the velocity of the shock (which is, thus, a non-classical shock, see LeFloch 2002). Whether or not such a simple approach can also be applied to our problem is currently an open question.

\section{Conclusions}

In this paper, we considered the solution of a model problem of miscible displacement between parallel plates in the absence of diffusion. By selecting a piecewise representation in the mobility-concentration dependence, the problem can also be considered as 'three-fluid' flow in the absence of capillarity. Assuming a 

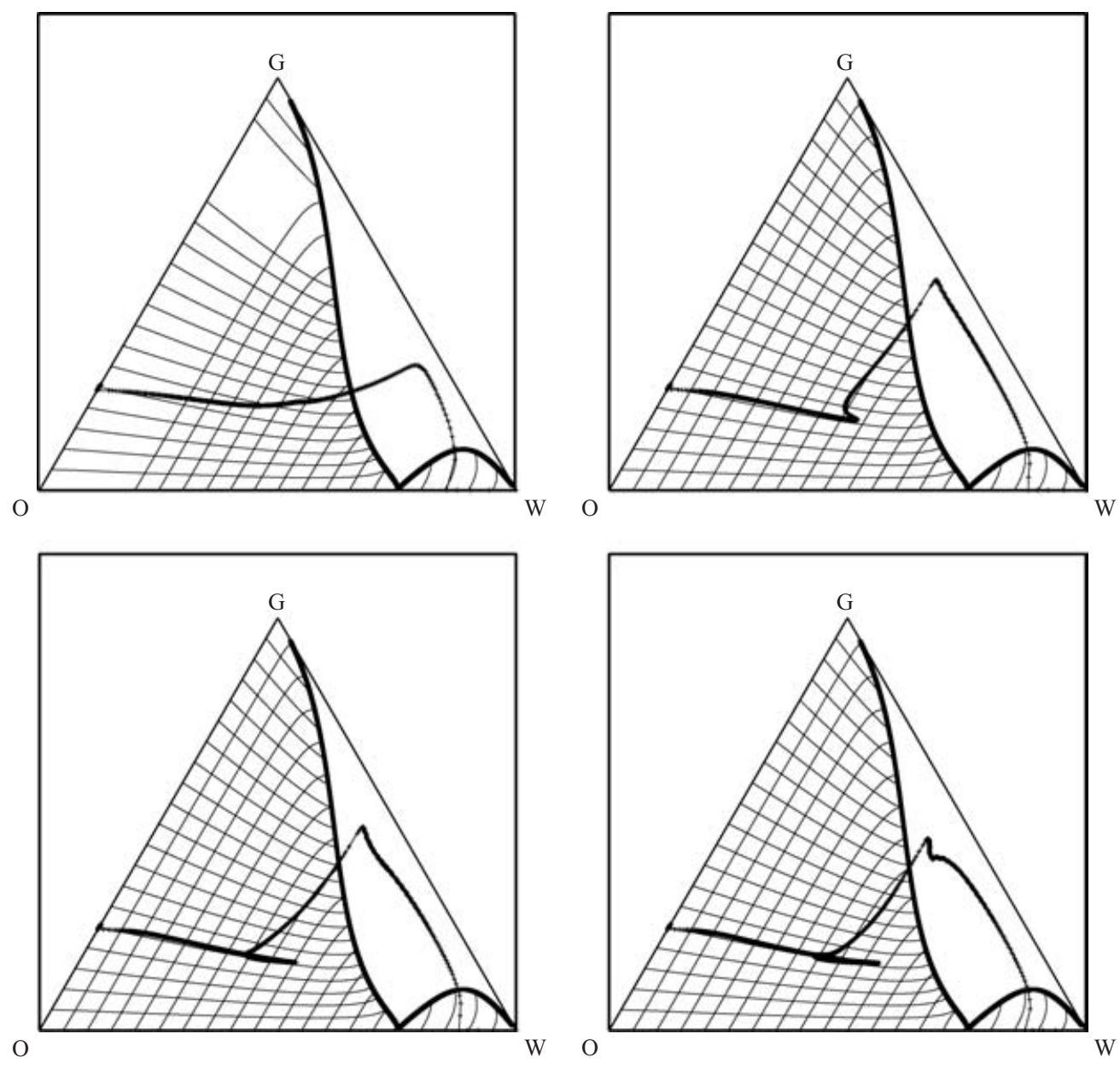

FIGURE 16. Consecutive snapshots in the triangular composition diagram of the solution corresponding to the conditions of figure 14 and obtained from the numerical simulations.

symmetric profile in the gap and based on a lubrication-type approximation, the mathematical description was formulated in terms of a set of two quasi-linear hyperbolic equations. Parallel flow is necessary for the consistency of the quasi-linear hyperbolic formalism at steady-state and represents the equivalent of the equilibrium approximation in other contexts.

We used this model to study the development of a mixed-type behaviour, namely of an elliptic region in the composition space. The system is hyperbolic and can be studied analytically, when the mobility profile is monotonic, or when the mobility of the middle phase is smaller than its neighbours. When the latter mobility is larger, a change of type is displayed and an elliptic region develops at the tip of the displacement. We studied analytically and numerically the conditions for the appearance of this behaviour.

Subsequently, the solution of typical Riemann problems was considered. Numerical solutions of the problem, under the parallel-flow assumption, but with small diffusion, based on lattice BGK simulations, were also obtained. Good agreement was found in the case where the solution is a rarefaction. When mixed-type behaviour develops, 

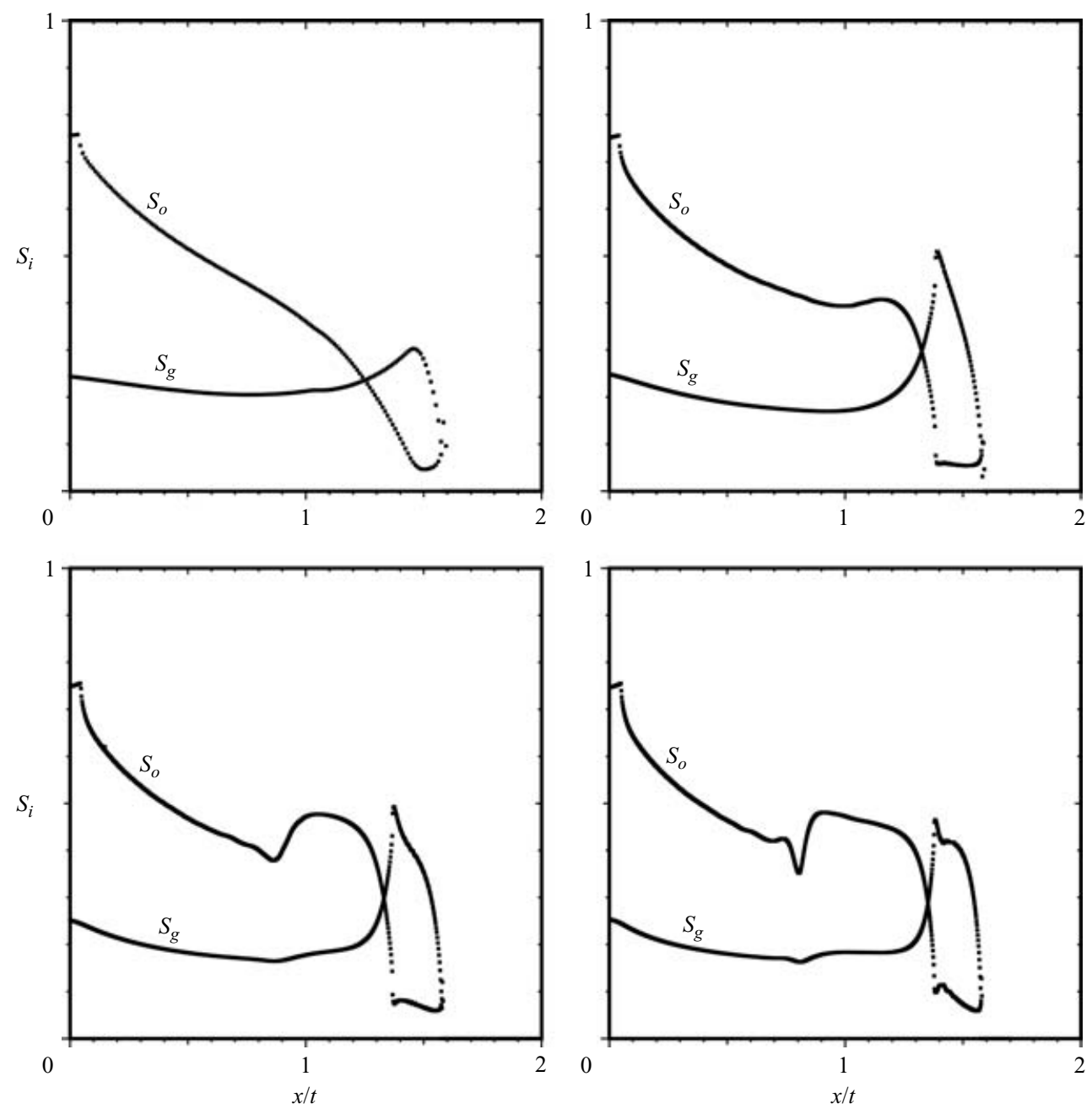

Figure 17. Consecutive snapshots of the volume fraction (saturation) profiles plotted as functions of the convective similarity variable $x / t$, corresponding to the conditions of figure 14 and obtained from the numerical simulations.

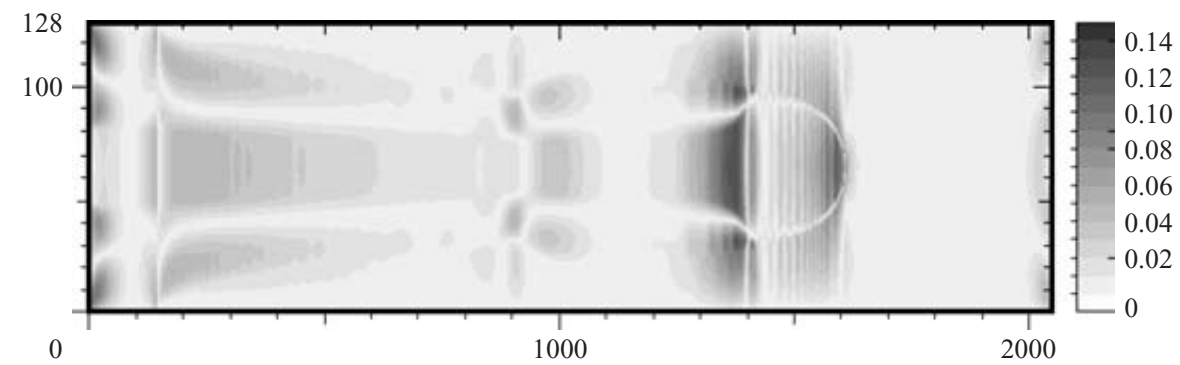

FIGURE 18. A snapshot of the difference between the actual values, obtained from the full simulation, and the lubrication (parallel flow) approximation expressions (8)-(9), of the velocity component in the direction of the displacement, for the conditions corresponding to figure 14 . Significant discrepancies exist near the tip (as well as near endpoints of the simulation). 
and diffusion is not strong enough, the solution path is forced to cross the elliptic region, and the solution becomes unstable, leading to unphysical oscillations. For the Riemann problems considered, the elliptic region arises precisely at the displacement front, where the parallel-flow approximation, underlying the quasi-linear hyperbolic formalism, fails. Crossing this region requires use of the full higher-dimensional model. The resulting structure at the tip cannot be captured by the quasi-linear hyperbolic formalism, regardless of the selection of the fractional flow expressions or the addition of regularizing diffusion. Rather, it necessitates incorporation of additional physics, which in the present paper were included by considering the higher-dimensional model. The solution obtained essentially corresponds to a non-linear eigenvalue problem for the shock velocity, and it is analogous to the non-classical shocks used for the resolution of corresponding problems in other contexts (e.g. see LeFloch 2002).

Our study shows that physically acceptable models can lead to change-of-type behaviour. Models are, therefore, not to be rejected, because they show the existence of an elliptic region. Rather, the emergence of a mixed-type region in such problems is a warning of the local failure of the equilibrium approximation, which in our context is the parallel-flow approximation, and which is fundamental for the development of the quasi-linear hyperbolic formalism in the first place. Crossing the elliptic region must be done by accounting for additional physics in that region. In the present paper, this necessitated consideration of the higher-dimensional model. Whether or not it is possible to simplify the description, for example, in terms of an equivalent capillary term, is currently under investigation. It is also worth noting that the appearance of a shock, the tip of which exceeded the maximum Poiseuille tip velocity of $3 / 2$, was associated in Lajeunesse et al. (1999) with the onset of instability in the third direction in the Hele-Shaw geometry (e.g. that which is perpendicular to the plane of the paper in figure 1). Whether or not this is also the case in the present problem when an elliptic region develops remains to be determined.

We close by adding that the parallel-flow approximation is also likely to fail in shocks, for example in the two-fluid problem of YY, or for appropriate Riemann problems in the hyperbolic region in the present three-fluid problem. Such shocks appear to be non-classical, and their resolution requires the consideration of additional physics and of an eigenvalue problem, much like that for crossing the elliptic region.

Many useful discussions with Professor Dan Marchesin and Dr C. Ruyer-Quil are gratefully acknowledged. This work was partly supported by IDRIS (project 034052). The PhD work of Laurent Talon is supported by a MRT grant. All these sources of support are gratefully acknowledged.

\section{REFERENCES}

Aldushin, A. P. \& Matkowsky, B. J. 1998 Instabilities, fingering and Saffman-Taylor problem in filtration combustion. Combust. Sci. Technol. 133, 293-341.

Barenblatt, G. I. 1996 Scaling, Self-Similarity and Intermediate Asymptotics. Cambridge Texts in Applied Mathematics, Cambridge University Press.

BAtchelor, G. K. \& JANSE VAN Rensburg, R. W. 1985 Structure formation in bidisperse sedimentation. J. Fluid Mech. 166, 379-407.

Bell, J. B., Trangenstein, J. A. \& Shubin, G. R. 1986 Conservation laws of mixed type describing three-phase flow in porous media. SIAM J. Appl. Maths 46, 1000-1023.

Chen, C. Y. \& Meiburg, E. 1996 Miscible displacements in capillary tubes. Part 2. Numerical simulations. J. Fluid Mech. 326, 57-65.

Courant, R. \& Friedrichs, K. O. 1948 Supersonic Flow and Shock Waves. Wiley-Interscience. 
Fayers, F. \& Matthews, J. 1984 Evaluation of normalized Stone's method for estimating three-phase relative permeabilities. Soc. Pet. Engng. J. 24, 225-232.

LeFloch, P. G. 2002 Hyperbolic Systems of Conservation Laws. Birkhauser.

IsaAcson, E., Marchesin, D. \& Plohr, B. J. 1990 Transitional waves for conservation laws. SIAM J. Appl. Math. Anal. 21, 837-866.

JACKsOn, M. D. \& BLunt, M. J. 2002 Elliptic region and stable solutions for three-phase flow in porous media. Transport in Porous Media 48, 249-269.

Juanes, R. \& Patzek, T. W. 2003 Analytical solution to the Riemann problem of three-phase flow in porous media. Transport in Porous Media 55, 47-70.

Keyfitz, B. L. 1989 Change of type in three-phase flow: a simple analog. J. Diff. Equations 80, $280-305$.

Lajeunesse, E., Martin, J., Rakotomalala, N., Salin, D. \& Yortsos, Y. C. 1999 Miscible displacements in a Hele-Shaw cell at high flow rates. J. Fluid Mech. 398, 299-319.

LaKe, L. W. 1989 Enhanced Oil Recovery. Prentice-Hall, Englewood Cliffs, NJ.

Loggia, D., Rakotomalala, N. \& Salin, D. 1999 The effect of mobility gradients on viscous instabilities in miscible flows in porous media. Phys. Fluids 11, 740-742.

Manickam, O. \& Homsy, G. M. 1993 Stability of miscible displacements in porous media with nonmonotonic viscosity profiles. Phys. Fluids A 5, 1356-1367.

Menikoff, R. \& Plohr, B. J. 1989 The Riemann problem for fluid flow of real materials. Rev. Mod. Phys. 61, 75-130.

Park, C. W. \& Homsy, G. M. 1984 Two-phase displacement in Hele-Shaw cells: theory. J. Fluid Mech. 139, 291-308.

Pelcé, P. 1988 Dynamics of Curved Fronts. Academic.

Petitjeans, P. \& Maxworthy, T. 1996 Miscible displacements in capillary tubes. Part 1. Experiments. J. Fluid Mech. 326, 37-56.

Rakotomalala, N., Salin, D. \& Watzky, P. 1997 Miscible displacement between two parallel plates: BGK lattice gas simulations. J. Fluid Mech. 338, 277-297.

Reinelt, D. A. \& Saffman, P. G. 1985 The penetration of a finger into a viscous fluid in a channel. SIAM J. Sci. Stat. Comput. 6, 542-561.

Saffman, P. G. \& TAYLOR, G. I. 1958 The penetration of a fluid into a porous medium or Hele-Shaw cell containing a more viscous liquid. Proc. R. Soc. Lond. A 245, 312-329.

Shariati, M. 2000 Effect of Non-Monotonic Mobility at Various Scales in Porous Media. PhD Dissertation, University of Southern California.

Shariati, M. \& Yortsos, Y. C. 2001 Stability of miscible displacements across stratified porous media. Phys. Fluids 13, 2245-2257.

Shearer, M. \& Trangenstein, J. 1989 Loss of real characteristics for models of three-phase flow in porous media. Transport Porous Media 4, 499-525.

SheARER, M. \& YANG, Y. 1995 The Riemann problem for a system of conservation laws of mixed type with a cubic nonlinearity. Proc. R. Soc. Edin. A 125, 675-699.

TruskinovsKy, L. 1987 Dynamics of nonequilibrium phase boundaries in a heat conducting elastic medium. Z. angew. Math. Mech. 51, 777-784.

Whitham, G. B. 1976 Linear and Nonlinear Waves. John Wiley.

YANG, Z. \& YorTsos, Y. C. 1997 Asymptotic solutions of miscible displacements in geometries of large aspect ratio. Phys. Fluids 9, 286-298. 